TRANSACTIONS OF THE

AMERICAN MATHEMATICAL SOCIETY

Volume 356, Number 11, Pages 4395-4425

S 0002-9947(04)03418-X

Article electronically published on January 23, 2004

\title{
ISOLATING BLOCKS NEAR THE COLLINEAR RELATIVE EQUILIBRIA OF THE THREE-BODY PROBLEM
}

\author{
RICHARD MOECKEL
}

\begin{abstract}
The collinear relative equilibrium solutions are among the few explicitly known periodic solutions of the Newtonian three-body problem. When the energy and angular momentum constants are varied slightly, these unstable periodic orbits become normally hyperbolic invariant spheres whose stable and unstable manifolds form separatrices in the integral manifolds. The goal of this paper is to construct simple isolating blocks for these invariant spheres analogous to those introduced by Conley in the restricted three-body problem. This allows continuation of the invariant set and the separatrices to energies and angular momenta far from those of the relative equilibrium.
\end{abstract}

\section{INTRODUCTION}

Lagrange constructed simple periodic solutions of the Newtonian three-body problem where the configuration rotates rigidly around the center of mass. For such a motion to be possible the masses must be arranged in a special shape called a central configuration. Either they must form an equilateral triangle or else they must be collinear with certain mutual distance ratios uniquely determined by the masses $m_{i}$ and the ordering of the bodies along the line.

Each central configuration actually gives rise to a two-parameter family of periodic orbits. By varying the size of the central configuration one finds a oneparameter family of rigidly rotating solutions. In addition, elliptical periodic motions are possible with eccentricities ranging from 0 (the rigidly rotating orbits) to 1 (homothetic collision orbits). Instead of the size and eccentricity of the orbits one could take the energy, $h$, and the angular momentum, $\Omega$, as parameters. For each fixed energy there will be a unique rigidly rotating solution, up to symmetry. This solution will have a definite value of $|\Omega|$. As the angular momentum is decreased, the orbits become elliptical with the eccentricity tending to 1 as $|\Omega| \rightarrow 0$.

It turns out that the elliptical periodic orbits for angular momenta just below that of the relative equilibria are part of a larger invariant set. In the planar case, this set is a normally hyperbolic sphere $S^{3}$ while in the spatial case it is an $S^{5}$. The existence of this invariant set follows from local calculations at the relative equilibria. However, from this kind of analysis it is difficult to determine for what range of parameters the set continues to exist.

A similar phenomenon occurs in the circular restricted three-body problem. In the planar case one finds an $S^{1}$, that is, a hyperbolic periodic orbit, but in the

Received by the editors December 11, 2002 and, in revised form, May 7, 2003.

2000 Mathematics Subject Classification. Primary 70F10, 70F15, 37N05.

Key words and phrases. Celestial mechanics, central configurations, three-body problem.

(C)2004 American Mathematical Society 
three-dimensional problem there is an $S^{3}$. For the planar case, Conley constructed a simple isolating block around the periodic orbit. This was extended to the spatial case by Appleyard [1]. The invariant three-sphere in the spatial problem and the isolating block around it were motivating examples for the abstract theory of isolating blocks and later for the Conley index theory. In this paper, similar blocks will be constructed for the planar and spatial unrestricted problems. In principle, this allows continuation of the invariant set to energies and angular momenta far from the relative equilibrium. In practice, it is still difficult to verify the required conditions, but some results can be obtained with the help of the computer.

Perhaps the most interesting feature of these invariant sets is that their stable and unstable manifolds have codimension one in the manifolds of fixed energy and angular momentum. For example, in the planar, unrestricted problem, the invariant three-sphere has four-dimensional stable and unstable manifolds while the integral manifold has dimension five. Although the continued invariant sets whose existence is proved here may not be smooth, normally hyperbolic spheres, the stable and unstable sets remain separatrices for all energies and angular momenta such that the isolating block exists.

\section{The REstricted PROBlem}

This section contains a description of the isolating blocks used by Conley for the planar and spatial restricted three-body problems. This provides good motivation for the approach used later for the unrestricted case. The original reference for this work is [4].

2.1. Equations of motion. The restricted three-body problem models the motion of a small mass under the influence of the gravitational forces of two larger masses called the primaries. The primaries, which have masses $m_{1}=1-\mu$ and $m_{2}=\mu$, are taken to move on circular orbits of the two-body problem, unaffected by the third mass. One can choose coordinates in $\mathbf{R}^{3}$ which rotate uniformly around the $z$-axis so that the primaries remain on the $x$-axis at positions $q_{1}=(-\mu, 0,0)$ and $q_{2}=(1-\mu, 0,0)$. Let $(x, y, z) \in \mathbf{R}^{3}$ and $(u, v, w) \in \mathbf{R}^{3}$ denote the position and velocity vectors of the third mass with respect to the rotating coordinate system. Then the equations of motion are:

$$
\begin{aligned}
\dot{x} & =u, & \dot{u} & =V_{x}-2 v, \\
\dot{y} & =v, & \dot{v} & =V_{y}+2 u, \\
\dot{z} & =w, & \dot{w} & =V_{z},
\end{aligned}
$$

where

$$
V(x, y, z)=\frac{1}{2}\left(x^{2}+y^{2}\right)+\frac{1-\mu}{r_{13}}+\frac{\mu}{r_{23}}
$$

and $r_{13}=\sqrt{(x+\mu)^{2}+y^{2}+z^{2}}, r_{23}=\sqrt{(x+\mu-1)^{2}+y^{2}+z^{2}}$ are the distances to the primaries. $V$ is just the Newtonian gravitational potential with an extra term representing the centrifugal force due to the rotating coordinates. Equations (2.1) define the spatial restricted three-body problem. The planar problem is obtained by restricting to the invariant set $\{z=w=0\}$. 
2.2. Integral manifolds and Hill's regions. The function

$$
H(x, y, z, u, v, w)=\frac{1}{2}\left(u^{2}+v^{2}+w^{2}\right)-V(x, y, z)
$$

is a constant of motion for (2.1). For any constant $\lambda \in \mathbf{R}$ let

$$
\begin{aligned}
\mathcal{M}(\lambda) & =\left\{(x, y, z, u, v, w) \in \mathbf{R}^{6}: H(x, y, z, u, v, w)=-\lambda\right\}, \\
\mathcal{H}(\lambda) & =\left\{(x, y, z) \in \mathbf{R}^{3}: \exists(u, v, w) \in \mathbf{R}^{3}, H(x, y, z, u, v, w)=-\lambda\right\} .
\end{aligned}
$$

$\mathcal{M}(\lambda)$ will be called an integral manifold and its projection onto the configuration space, $\mathcal{H}(\lambda)$, is the corresponding Hill's region. In this paper, the total energy $H$ is always negative and so $\lambda$ is always positive.

For points in $\mathcal{M}(\lambda)$ one has $V(x, y, z)-\lambda=\frac{1}{2}\left(u^{2}+v^{2}+w^{2}\right) \geq 0$. In fact, this inequality characterizes the Hill's region and so

$$
\mathcal{H}(\lambda)=\left\{(x, y, z) \in \mathbf{R}^{3}: V(x, y, z) \geq \lambda\right\} .
$$

The Hill's regions for the planar problem can be visualized by making a contour plot of $V(x, y, 0)$ (see Figure 1). The shaded region represents a value of $\lambda$ typical of those considered below.

For the spatial problem, it is necessary to plot level surfaces of $V(x, y, z)$. For the value of $\lambda$ shaded in the figure, the corresponding three-dimensional Hill's region resembles the surface obtained by rotating the two-dimensional region around the $x$-axis (but it is not really a surface of revolution).

For each fixed $(x, y, z)$ in the interior of $\mathcal{H}(\lambda)$, the equation $H=-\lambda$ defines a two-sphere in $(u, v, w)$-space. On the boundary of $\mathcal{H}(\lambda)$, the radius of the sphere becomes zero and the sphere becomes a point. The points $q_{1}=(1-\mu, 0,0)$ and $q_{2}=(\mu, 0,0)$, where the primaries are located, are singular points of the function $V(x, y, z)$ and so must be excluded from $\mathcal{H}(\lambda)$. In the planar case, one has a circle in the $(u, v)$-plane instead of a two-sphere.

From these observations one can work out the topological structure of the integral manifolds $\mathcal{M}(\lambda)$. For example, the integral manifold of the planar problem corresponding to the shaded figure-eight part of Hill's region in Figure 1 is diffeomorphic to a three-sphere with two unlinked circles deleted. To see this, note that the Hill's region is a two-disk with two deleted points. Over each point of this punctured two-disk lies a circle in the $(u, v)$-plane, except that over the boundary circle of the two-disk the circles collapse to points. Except for the punctures, this description applies equally well to the standard three-sphere in $\mathbf{R}^{4}$ relative to its projection onto a coordinate plane. Now it is not difficult to construct the required diffeomorphism. In the spatial problem for the same value of $\lambda$ one finds that $\mathcal{M}(\lambda)$ is a five-dimensional sphere with two unlinked two-spheres deleted.

2.3. The Lagrange point $L_{2}$. The critical points of $V(x, y, z)$ are called the $L a$ grange points. They determine equilibrium points of the differential equations in rotating coordinates. In non-rotating coordinates, they determine periodic solutions for which the configuration rotates rigidly at constant speed.

One finds

$$
\begin{aligned}
& V_{x}=x-\gamma^{2} x+(1-\mu) \mu\left(\frac{1}{r_{23}^{3}}-\frac{1}{r_{13}^{3}}\right), \\
& V_{y}=y-\gamma^{2} y \\
& V_{z}=-\gamma^{2} z
\end{aligned}
$$



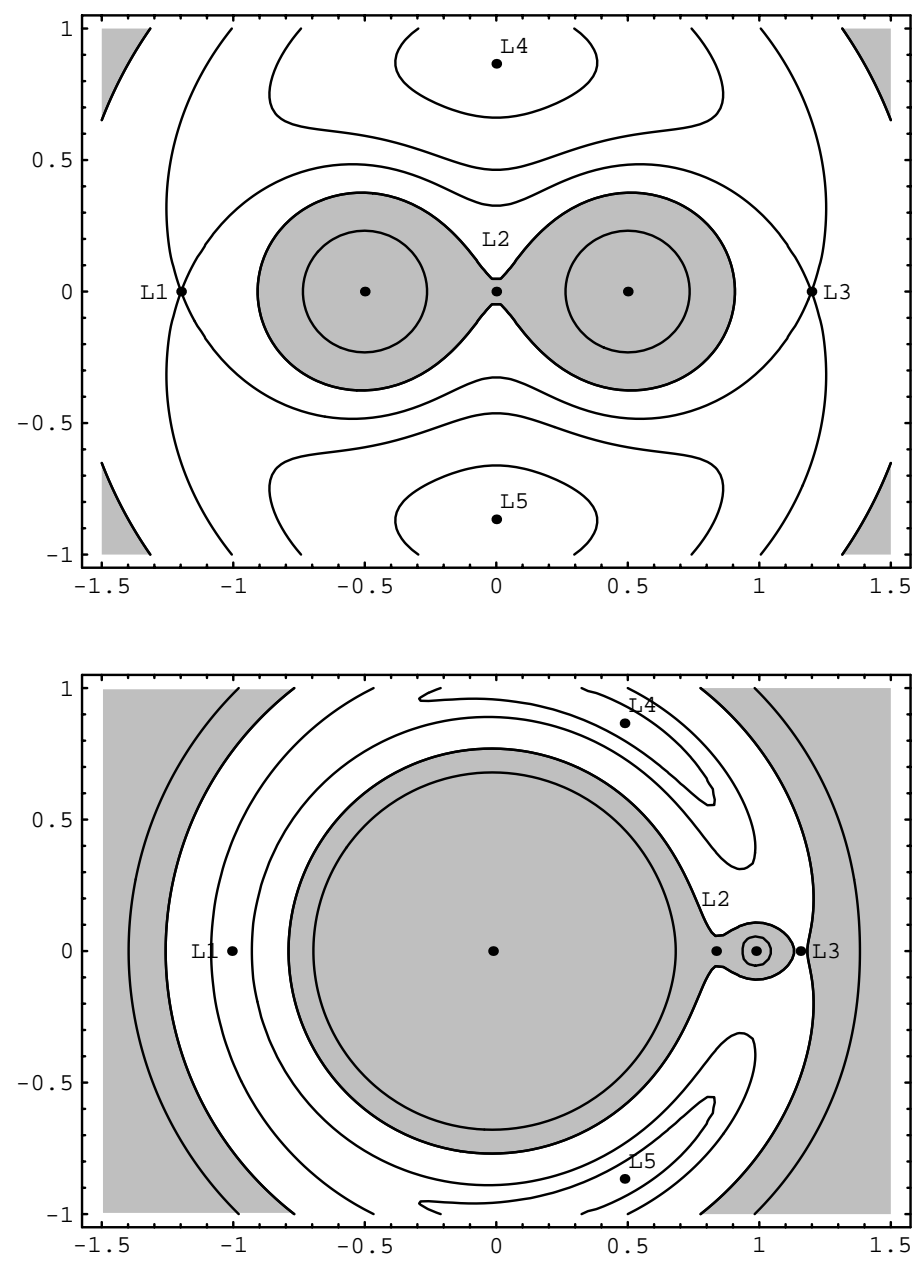

FiguRE 1. Hill's regions and Lagrange points for the planar restricted three-body problems with $\mu=\frac{1}{2}$ (top) and $\mu=\frac{1}{81}$ (bottom). The latter is called the lunar problem since $\mu$ approximates the mass ratio of the moon and earth.

where

$$
\gamma^{2}=\frac{1-\mu}{r_{13}^{3}}+\frac{\mu}{r_{23}^{3}}
$$

The equation $V_{z}=0$ implies $z=0$ so all the critical points are planar. There are five well-known critical points for the planar potential $V(x, y, 0)$ all of which are visible in Figure 1 The points $L_{4}$ and $L_{5}$ correspond to equilateral triangle configurations. The other three critical points lie on the $x$-axis and so represent collinear configurations. These are critical points of the collinear potential $V(x, 0,0)$. 


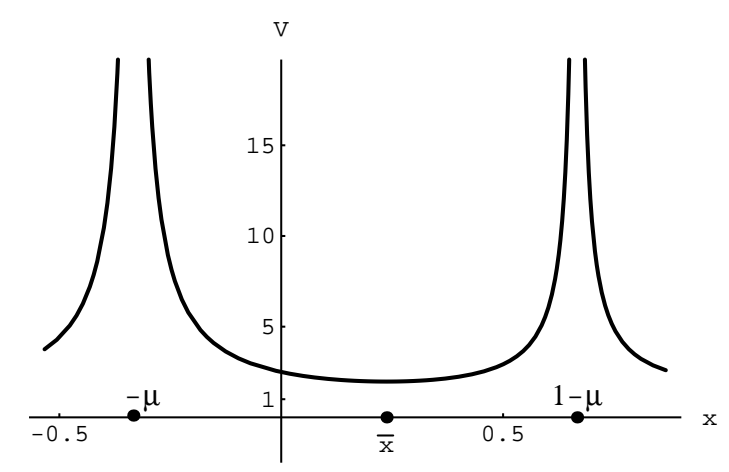

Figure 2. The collinear potential. The critical point $\bar{x}$ of this convex function is the Lagrange point $L_{2}$.

Figure 2 shows the part of the graph of $V(x, 0,0)$ between the primaries. One finds

$$
V_{x x}(x, 0,0)=1+2 \gamma^{2}>0
$$

so $V(x, 0,0)$ is convex. This shows that there is a unique critical point $L_{2}=$ $(\bar{x}, 0,0)$ in the interval. The exact position of the critical point depends on $\mu$ in a complicated way, but there is a simple argument to show that $\bar{x}$ is always closer to the smaller of the two primaries. Just note that at the midpoint, $x=\frac{1}{2}-\mu$, of the primaries, $r_{13}=r_{23}=\frac{1}{2}$. A short computation gives $V_{x}\left(\frac{1}{2}-\mu, 0,0\right)=7\left(\mu-\frac{1}{2}\right)$. If $\mu<\frac{1}{2}$, then $V_{x}<0$ at the midpoint and the convexity implies that the critical point is to the right of the midpoint. Similarly, if $\mu>\frac{1}{2}$, the critical point is to the left of the midpoint. This observation can be used to estimate $\gamma^{2}$ at the critical point. Just write the equation $V_{x}=0$ in the form $\left(\gamma^{2}-1\right) \bar{x}=(1-\mu) \mu\left(r_{23}^{-3}-r_{13}^{-3}\right)$. If $\mu<\frac{1}{2}$, then by the argument above, the right side is positive at $\bar{x}$. But in this case $\bar{x}$ is also positive and hence $\gamma^{2}>1$. If $\mu>\frac{1}{2}$, one finds the same result.

Let the value of the integral at $L_{2}$ be $H(\bar{x}, 0,0,0,0,0)=-V(\bar{x}, 0,0)=-\bar{\lambda}$. The critical integral "manifold" $\mathcal{M}(\bar{\lambda})$ is actually not a manifold near the critical point. But the nearby integral manifolds $\mathcal{M}(\lambda), \lambda<\bar{\lambda}$, are non-critical. The shaded Hill's region in Figure 1 corresponds to such a value of $\lambda$. Of course these nearby manifolds do not contain the equilibrium point. Instead they contain a compact invariant set of orbits which remain for all time near $L_{2}$ but are not equilibria.

For $\lambda-\bar{\lambda}<0$ and small, one can investigate this invariant set by local methods. First, consider the linearization of the diffential equations near the equilibrium. The $6 \times 6$ linearized system can be split into $4 \times 4$ and $2 \times 2$ blocks corresponding to the $(x, y, u, v)$ and $(z, w)$ variables, respectively. The matrices are

$$
A=\left[\begin{array}{cccc}
0 & 0 & 1 & 0 \\
0 & 0 & 0 & 1 \\
1+2 \gamma^{2} & 0 & 0 & -2 \\
0 & 1-\gamma^{2} & 2 & 0
\end{array}\right], \quad B=\left[\begin{array}{cc}
0 & 1 \\
-\gamma^{2} & 0
\end{array}\right]
$$

The characteristic polynomials can be written as

$$
P_{A}(\zeta)=\zeta^{2}+\left(2-\gamma^{2}\right) \zeta-\left(\gamma^{2}-1\right)\left(2 \gamma^{2}+1\right), \quad P_{B}(\zeta)=\zeta+\gamma^{2},
$$


where $\zeta$ represents the square of an eigenvalue. Since $\gamma^{2}>1$ at $L_{2}$ for all values of the paremeter $\mu, A$ always has two real eigenvalues $\pm \alpha$ and two imaginary eigenvalues $\pm i \beta$ while $B$ has eigenvalues $\pm i \gamma$.

Some computations involving the eigenvectors show that the Hessian $D^{2} H$ at $L_{2}$ is positive definite on the direct sum of the invariant subspaces corresponding to the four imaginary eigenvalues $\pm i \beta, \pm i \gamma$. Thus using the linearized system and the quadratic part of $H$, the nearby integral manifolds $\mathcal{M}(\lambda), \lambda<\bar{\lambda}$, contain three-dimensional spheres filled with periodic and quasi-periodic solutions. The remaining two dimensions of phase space are hyperbolic with one stable and one unstable eigenvalue. By using the center manifold theorem one can show that these invariant three-spheres actually exist for the nonlinear system.

In the planar problem, there is only one pair of imaginary eigenvalues $\pm i \beta$, and a similar analysis implies the existence of a normally hyperbolic invariant circle, i.e., a hyperbolic periodic orbit, on the nearby integral manifolds. This could also be shown by invoking the Lyapunov center theorem. This planar periodic solution is one of the orbits in the invariant three-sphere for the spatial problem. Another application of the Lyapunov center theorem gives a second periodic solution corresponding to the imaginary eigenvalues $\pm i \gamma$. The motion of the small mass along this orbit is approximately orthogonal to the plane of the primaries.

2.4. The isolating block. Conley's approach to the local dynamics near $L_{2}$ was quite different [4]. For the planar case, he considered neighborhoods of $L_{2}$ in the Hill's regions, $\mathcal{H}(\lambda)$, of the form $a \leq x \leq b$, where the interval $(a, b)$ contains the critical point $\bar{x}$. These neighborhoods form "tunnels" between the two lobes of the Hill's region as illustrated in Figure 3. The preimage of these tunnels in the integral manifold $\mathcal{M}(\lambda)$ will be denoted by $B_{\lambda}(a, b)$. Conley showed that, at least for $\lambda-\bar{\lambda}$ sufficiently small, the sets $B_{\lambda}$ are manifolds-with-boundary which are convex to the flow. This means that any orbit which meets one of the boundary manifolds where $x=a$ or $x=b$ tangentially must lie outside of $B_{\lambda}$ both before and after the encounter. In later work with Easton, such submanifolds were called isolating blocks and the maximal invariant sets inside them were called isolated invariant sets. The external tangency condition ensures that the boundary points of the block are not part of the maximal invariant set in the block, so the invariant set is in the interior of the block. The analogous blocks for the spatial case were first considered by Appleyard [1].

It is easy to formulate the isolating block condition for $B_{\lambda}$ using second derivatives. One requires that any orbit with $x=a$ and $\dot{x}=0$ must also have $\ddot{x}<0$ and similarly that whenever $x=b$ and $\dot{x}=0$ one must have $\ddot{x}>0$. Now

$$
\begin{aligned}
& \dot{x}=u, \\
& \ddot{x}=\dot{u}=V_{x}-2 v .
\end{aligned}
$$

So the isolating block condition on the right boundary amounts to showing that $V_{x}>2 v$ for every point $(b, y, z, 0, v, w) \in \mathcal{M}(\lambda)$. From the convexity of $V(x, 0,0)$ it follows that $V_{x}(b, 0,0)>0$. Also, the equation $H=-\lambda$ implies that $v^{2} \leq 2(V-\lambda)$. Thus it suffices to show that

$$
V_{x}^{2}(x, y, z)>8(V(x, y, z)-\lambda)
$$

holds for all points $(x, y, z) \in \mathcal{H}_{l}$ with $x=b$. A similar analysis at $x=a$ yields the same inequality. 


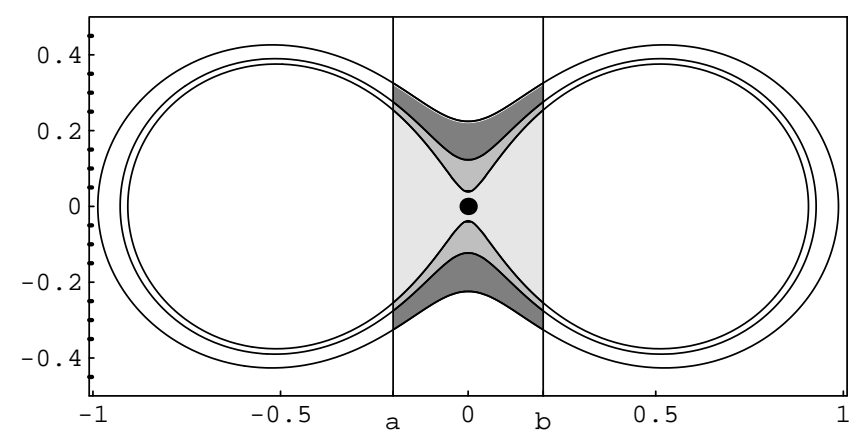

FIgURE 3. Projections onto the configuration space of the isolating blocks $B_{\lambda}(a, b)$ for three values of $\lambda$.

Inequality (2.4) can be verified for $\lambda-\bar{\lambda}<0$ and small as follows. Taylor expansion of $V(x, y, z)$ near $L_{2}$ gives

$$
V(\bar{x}+\delta x, y, z)=\bar{\lambda}+\frac{1}{2}\left(\left(1+2 \gamma^{2}\right)(\delta x)^{2}-\left(\gamma^{2}-1\right) y^{2}-\gamma^{2} z^{2}\right)+\ldots
$$

and so $V_{x}(\bar{x}+\delta x, y, z)=\left(1+2 \gamma^{2}\right) \delta x+\ldots$. Now introduce scaled variables by setting

$$
\delta x=\epsilon \xi, \quad y=\epsilon \eta, \quad z=\epsilon \zeta, \quad \lambda=\bar{\lambda}-\epsilon^{2},
$$

where $\epsilon>0$ is a small parameter. The boundary of Hill's region $\mathcal{H}(\lambda)$ is approximately the hyperboloid

$$
\left(1+2 \gamma^{2}\right) \xi^{2}-\left(\gamma^{2}-1\right) \eta^{2}-\gamma^{2} \zeta^{2}=2
$$

and inequality (2.4) is approximately

$$
\left(1+2 \gamma^{2}\right)^{2} \xi^{2}>4\left(1+2 \gamma^{2}\right) \xi^{2}+8-4\left(\left(\gamma^{2}-1\right) \eta^{2}+\gamma^{2} \zeta^{2}\right)>4\left(1+2 \gamma^{2}\right) \xi^{2}+8 .
$$

If one can find $\xi$ such that $\left(1+2 \gamma^{2}\right)\left(2 \gamma^{2}-3\right) \xi^{2}>8$, then setting $a=\bar{x}-\epsilon \xi, b=\bar{x}+\epsilon \xi$ will yield an isolating block for all sufficiently small $\epsilon$. Clearly this is possible if and only if $\gamma^{2}>\frac{3}{2}$. It turns out that this inequality holds for all $\mu$ and so one can always construct the isolating blocks. This is Conley's result in [4].

These small isolating blocks, which exist for values of $\lambda$ close to that of the Lagrange point, will be called infinitesimal isolating blocks. One can also try to analyze inequality (2.4) for more general values of $a, b, \lambda$. This can be done in some cases but the inequality is complicated enough to require some computer-assisted computations. For example, in the cases $\mu=\frac{1}{2}$ and $\mu=\frac{1}{81}$, the parameter values $(c, \lambda)$ for which the isolating block inequality is satisfied on the segment $x=c$ is shown in Figure 4. Fixing a value of $c$ one finds an interval of values of the integral $\lambda$ for which the set remains an isolating block. Similarly, fixing $\lambda$ gives an interval of possible choices for the position, $c$, of each wall of the block. See section 3.5 for a discussion of how the analogous figures for the unrestricted problem are computed.

Let $S_{\lambda}(a, b)$ denote the maximal invariant set in the block $B_{\lambda}(a, b)$. This will be some compact invariant set contained in the interior of $B_{\lambda}$. For $\lambda$ close enough to the critical value $\bar{\lambda}$ and for $a, b$ sufficiently close to the Lagrange point, the linearized differential equation can be used to show that $S_{\lambda}(a, b)$ is the normally hyperbolic periodic orbit (planar problem) or the invariant three-sphere (spatial problem) mentioned above. From the fact that $S_{\lambda}(a, b)$ is contained in the interior of $B_{\lambda}(a, b)$, it follows that $S_{\lambda}(a, b)$ does not change as the values of $a, b$ are varied 

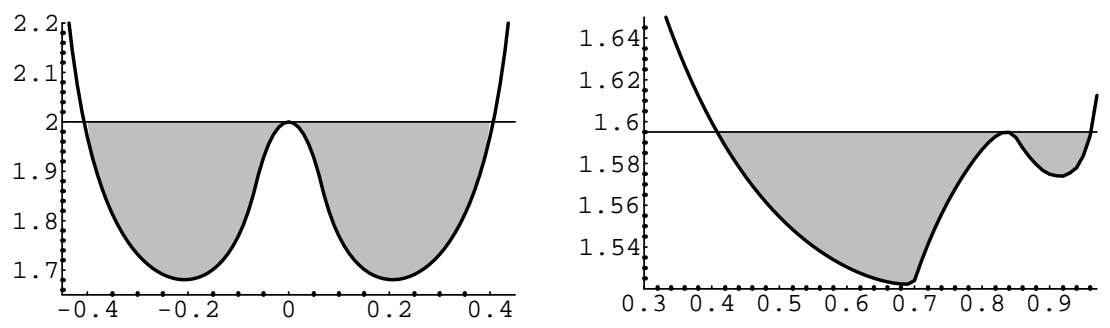

FigURE 4. Isolating block bifurcation diagrams for equal primaries $\mu=\frac{1}{2}$ (left) and the "lunar problem" $\mu=\frac{1}{81}$ (right). Horizontal axis shows that $x=c$ and that the vertical axis is the integral parameter, $\lambda$. The horizontal line shows the critical level $\lambda=\bar{\lambda}$.

over the intervals of values for which the isolating block inequality holds. So even for quite large isolating blocks, the invariant set is still just the periodic orbit or invariant sphere. But it is not clear how small $\bar{\lambda}-\lambda$ must be for this characterization to hold.

For values of $\lambda$ far from $\bar{\lambda}$, one can use the topology of the block $B_{\lambda}$ to get some information about the invariant set. First, the Conley index theory implies that the invariant set remains nonempty [5], 6]. Indeed, this topological index is invariant under continuation so the unknown invariant set has the same index as the normally hyperbolic periodic orbit or sphere, and these are known to be nontrivial. Stronger conclusions can be obtained using the fact that the exit set of the isolating block has two components. The exit set of an isolating block is simply the set of boundary points of the block at which orbits are leaving in forward time. For $B_{\lambda}(a, b)$, this is just the union of the set with $x=b$ and $\dot{x} \geq 0$ and the set with $x=a$ and $\dot{x} \leq 0$. For the spatial restricted problem, each of the boundary components $x=a, b$ is a four-dimensional sphere and the exit set consists of two four-dimensional disks, one in each boundary component. Now one of the most important consequences of the convexity to the flow is that the time $T(p)$ required for a point $p \in B$ to reach the exit set depends continously on $p$ [7]. More precisely, if one defines the stable set $S_{\lambda}^{+}=\left\{p: \phi_{t}(p) \in B_{\lambda}, t \geq 0\right\}$ to be the set of points whose forward orbits remain in $B_{\lambda}$, then $T: B_{\lambda} \backslash S_{\lambda}^{+} \rightarrow R^{+}$is continuous. It follows that the set $S_{\lambda}^{+}$is a separatrix, i.e., $B_{\lambda} \backslash S_{\lambda}^{+}$is disconnected. To see this, note that the continuity of exit time $T(p)$ implies the continuity of the exit point $\phi_{T(p)}(p)$. So the decomposition of $B_{\lambda} \backslash S_{\lambda}^{+}$ into the points which exit through $x=a$ and the points which exit through $x=b$ is a separation into disjoint, nonempty open sets. This means that although the structure of the invariant set may change with $\lambda$, its stable set remains a separatrix for the flow as long as the isolating block continues to exist.

The existence of a separatrix is perhaps the most important conclusion following from the construction of the isolating block. However, with a little more information about the flow, a theorem of Easton allows one to show that the invariant set itself retains some of the character of the three-sphere. For parameter values $\lambda$ close to $\bar{\lambda}$, Conley completely analyzed the flow through his infinitesimal isolating blocks [4]. His main interest was the investigation of transit orbits, i.e., orbits entering the block at one of the boundary spheres and exiting at the other. For the infinitesimal block, transit orbits from $x=a$ to $x=b$ and from $x=b$ to $x=a$ exist. Nontransit 
orbits also exist. For example, it follows from continuity of the flow that near any orbit which meets $x=b, \dot{x}=0, \ddot{x}>0$, there are orbits with $x=b, \dot{x}<0$ which enter $B$ and then quickly leave through the same boundary component. Now Easton's result shows that the existence of these types of orbits implies that the invariant set inside the block has a nonzero three-dimensional Cech cohomology (like the three-sphere) [7. It follows from this that its topological dimension is at least three. To apply the theorem one must prove the existence of both types of transit orbits and both types of nontransit orbits. The argument above shows that the nontransit orbits exist for $B_{\lambda}(a, b)$ whenever the convexity condition holds. However, it seems difficult to rigorously prove the existence of the transit orbits when $\bar{\lambda}-\lambda$ is large. Nevertheless one can easily find them numerically (Figure 9 shows a transit orbit for the unrestricted, planar problem). The next section is devoted to the construction of similar isolating blocks for the planar and spatial unrestricted three-body problems. Once they are shown to exist, the existence of a separatrix follows by the same argument used here.

The existence of the separatrix for the restricted problem has been crucial in some recent applications 22], 9], [12. It separates the transit orbits passing through the tunnel near the Lagrange point from the non-transit orbits which turn around in the tunnel and return to the same lobe of the Hill's region from which they came. To go through the tunnel, choose an initial condition on the transit side of the separatrix. On the other hand, if one wants to "park" near the Lagrange point, the initial condition can be taken on the separatrix.

\section{THE UNRESTRICTED THREE-BODY PROBLEM}

Now consider the problem of three masses of comparable size moving under the influence of their mutual gravitational forces. Many of the features of the restricted problem carry over to this case. There are analogues of the Hill's regions, the Lagrange equilibrium points and the invariant spheres nearby. The main goal of this paper is to show that, with some restrictions on the masses, there are also isolating blocks like those described above. From this one can deduce the existence of separatrices of the unrestricted three-body problem by the same argument outlined above.

3.1. Equations of motion. Both Cartesian and Jacobi coordinates will be used.

3.1.1. Cartesian coordinates. Let $m_{i}>0$ be the masses and $q_{i} \in \mathbf{R}^{d}$ be the positions of the bodies, $i=1,2,3$. Here $d=2$ for the planar three-body problem and $d=3$ for the spatial problem. Let $r_{i j}=\left|q_{i}-q_{j}\right|$ denote the mutual distances between the mass points and define the Newtonian potential function

$$
U\left(q_{1}, q_{2}, q_{3}\right)=\frac{m_{1} m_{2}}{r_{12}}+\frac{m_{1} m_{3}}{r_{13}}+\frac{m_{2} m_{3}}{r_{23}} .
$$

Newton's equations of motion are

$$
m_{i} \ddot{q}_{i}=U_{q_{i}}, \quad i=1,2,3,
$$

where the $U_{q_{i}}$ denotes the $d$-dimensional vector of partial derivatives of $U$ with respect to the components of $q_{i}$. If one sets $q=\left(q_{1}, q_{2}, q_{3}\right) \in \mathbf{R}^{3 d}$ and defines a $3 d \times 3 d$ mass matrix $M=\operatorname{diag}\left(m_{1}, \ldots, m_{3}\right)$, then Newton's equations are simply

$$
M \ddot{q}=\nabla U(q) .
$$


Define the kinetic energy

$$
T=\frac{1}{2}\left(m_{1}\left|\dot{q}_{1}\right|^{2}+m_{2}\left|\dot{q}_{2}\right|^{2}+m_{3}\left|\dot{q}_{3}\right|^{2}\right)=\frac{1}{2} \dot{q} \cdot M \dot{q}
$$

and the Lagrangian

$$
L=T+U \text {. }
$$

Then Newton's equations are just the Euler-Lagrange equations

$$
\dot{L}_{\dot{q}}=L_{q} .
$$

The constants of motions of the system are the linear momentum vector

$$
m_{1} q_{1}+m_{2} q_{2}+m_{3} q_{3}
$$

the angular momentum vector

$$
\Omega=m_{1} q_{1} \times \dot{q}_{1}+m_{2} q_{2} \times \dot{q}_{2}+m_{3} q_{3} \times \dot{q}_{3}
$$

and the total energy

$$
H=T-U \text {. }
$$

3.1.2. Jacobi coordinates. Changing to Jacobi coordinates makes it easy to eliminate the center of mass and reduce the dimension of the system. First define several mass parameters

$$
\begin{aligned}
m_{12}=m_{1}+m_{2}, & m=m_{1}+m_{2}+m_{3}, \\
\nu_{1}=\frac{m_{1}}{m_{12}}, & \nu_{2}=\frac{m_{2}}{m_{12}}, \\
\mu_{1}=\frac{m_{1} m_{2}}{m_{12}}, & \mu_{2}=\frac{m_{12} m_{3}}{m}
\end{aligned}
$$

and centers of mass

$$
c_{12}=\frac{m_{1} q_{1}+m_{2} q_{2}}{m_{12}}, \quad c=\frac{m_{1} q_{1}+m_{2} q_{2}+m_{3} q_{3}}{m} .
$$

Then the Jacobi coordinates are $x=q_{2}-q_{1}, y=q_{3}-c_{12}$ and $c$. This is a linear change of variables

$$
\left[\begin{array}{l}
x \\
y \\
c
\end{array}\right]=\left[\begin{array}{ccc}
-1 & 1 & 0 \\
-\nu_{1} & -\nu_{2} & 1 \\
\frac{m_{1}}{m} & \frac{m_{2}}{m} & \frac{m_{3}}{m}
\end{array}\right]\left[\begin{array}{l}
q_{1} \\
q_{2} \\
q_{3}
\end{array}\right], \quad\left[\begin{array}{l}
q_{1} \\
q_{2} \\
q_{3}
\end{array}\right]=\left[\begin{array}{ccc}
-\nu_{2} & -\frac{m_{3}}{m} & 1 \\
\nu_{1} & -\frac{m_{3}}{m} & 1 \\
0 & \frac{m_{12}}{m} & 1
\end{array}\right]\left[\begin{array}{l}
x \\
y \\
c
\end{array}\right] .
$$

Making these substitutions in the kinetic and potential energies gives

$$
\begin{aligned}
T & =\frac{1}{2}\left(\mu_{1}|\dot{x}|^{2}+\mu_{2}|\dot{y}|^{2}+m|\dot{c}|^{2}\right), \\
U(x, y) & =\frac{m_{1} m_{2}}{|x|}+\frac{m_{1} m_{3}}{\left|y+\nu_{2} x\right|}+\frac{m_{2} m_{3}}{\left|y-\nu_{1} x\right|} .
\end{aligned}
$$

As before, $L=T-U$ and the equations of motion are the Euler-Lagrange equations.

In particular, the equation $\dot{L}_{\dot{c}}=L_{c}$ for the center of mass gives $m \ddot{c}=0$ which shows that the linear momentum vector $m \dot{c}$ is constant. Assuming without loss of generality that $m \dot{c}=0$, the center of mass $c$ will also be constant and it may be assumed that $c=0$. This leaves only the variables $x, y$ which satisfy the first-order system

$$
\begin{array}{lc}
\dot{x}=u, & \dot{u}=\frac{1}{\mu_{1}} U_{x}, \\
\dot{y}=w, & \dot{w}=\frac{1}{\mu_{2}} U_{y} .
\end{array}
$$


The remaining constants of motion are the angular momentum vector and the energy:

$$
\begin{aligned}
& \Omega=\mu_{1} x \times u+\mu_{2} y \times w, \\
& H=\frac{1}{2}\left(\mu_{1}|u|^{2}+\mu_{2}|w|^{2}\right)-U(x, y) .
\end{aligned}
$$

3.2. Integral manifolds and Hill's regions. Fixing the energy and the angular momentum vector gives an invariant manifold for the three-body problem. However, the geometry is simpler in the spatial case if one fixes only the total angular momentum $|\Omega|$. The spatial case will be treated first and then the modifications needed for the planar case will be described. For any constants $h, \omega \in \mathbf{R}$ let

$$
\begin{aligned}
\mathcal{M}(h, \omega) & =\left\{(x, y, u, w) \in \mathbf{R}^{12}: H=h,|\Omega|=\omega\right\}, \\
\mathcal{H}(h, \omega) & =\left\{(x, y) \in \mathbf{R}^{6}: \exists(u, w) \in \mathbf{R}^{6}, H=h,|\Omega|=\omega\right\} .
\end{aligned}
$$

As before, $\mathcal{M}(h, \omega)$ will be called an integral manifold and $\mathcal{H}(h, \omega)$ the corresponding Hill's region.

To describe the Hill's regions, it is convenient to introduce new variables $(r, \xi, \eta)$, where

$$
r^{2}=\mu_{1}|x|^{2}+\mu_{2}|y|^{2}, \quad \xi=\frac{x}{r}, \quad \eta=\frac{y}{r} .
$$

The variable $r$ is the square root of the moment of inertia of the configuration with respect to the origin. It provides a natural measure of the size of the configuration formed by the three masses. The normalized configuration $(\xi, \eta)$ satisfies

$$
\mu_{1}|\xi|^{2}+\mu_{2}|\eta|^{2}=1 \text {. }
$$

$(\xi, \eta)$ describes the shape and orientation of the triangle formed by the three masses without regard to size. The five-dimensional ellipsoid

$$
\Sigma=\left\{(x, y) \in \mathbf{R}^{6}: \mu_{1}|x|^{2}+\mu_{2}|y|^{2}=1\right\}
$$

will be called the normalized configuration space.

Every configuration $(x, y) \in \mathbf{R}^{6} \backslash(0,0)$ determines a unique normalized configuration $(\xi, \eta) \in \Sigma$ where $(x, y)=(r \xi, r \eta)$. Denote the restriction of the Newtonian potential to $\Sigma$ by $U(\xi, \eta)$. Then $U(\xi, \eta)=r U(x, y)$ and the angular momentum and energy can be written as

$$
\begin{aligned}
& \Omega=r\left(\mu_{1} \xi \times u+\mu_{2} \eta \times w\right), \\
& H=\frac{1}{2}\left(\mu_{1}|u|^{2}+\mu_{2}|w|^{2}\right)-\frac{1}{r} U(\xi, \eta) .
\end{aligned}
$$

In addition to the Hill's regions, define the normalized Hill's region

$$
\mathcal{H}_{1}(h, \omega)=\left\{(\xi, \eta) \in \Sigma: \exists(r, u, w) \in \mathbf{R}^{+} \times \mathbf{R}^{6}, H=h,|\Omega|=\omega\right\}
$$

where $H, \Omega$ are given by (3.6). Then

Proposition 3.1. If $h<0$ and $\omega>0$, the Hill's regions and normalized Hill's regions can be characterized as follows:

$$
\begin{aligned}
\mathcal{H}(h, \omega) & =\left\{(x, y) \in \mathbf{R}^{6}: U(x, y) \geq|h|+\frac{\omega^{2}}{2 r^{2}}\right\}, \\
\mathcal{H}_{1}(h, \omega) & =\{(\xi, \eta) \in \Sigma: U(\xi, \eta) \geq \lambda\}
\end{aligned}
$$

where $\lambda=\sqrt{2|h| \omega^{2}}$. 
Proof. If $(x, y) \in \mathcal{H}(h, \omega)$, then there is some $(u, w) \in \mathbf{R}^{6}$ for which $H(x, y, u, w)=$ $h$. Since $h<0$ this implies

$$
U(x, y)=|h|+\frac{1}{2}\left(\mu_{1}|u|^{2}+\mu_{2}|w|^{2}\right) .
$$

Now $(x, y, u, w)$ must also satisfy $|\Omega|=\omega$ and so

$$
\omega^{2}=\left|\mu_{1} x \times u+\mu_{2} y \times w\right|^{2} \leq\left(\mu_{1}|x|^{2}+\mu_{2}|y|^{2}\right)\left(\mu_{1}|u|^{2}+\mu_{2}|w|^{2}\right) .
$$

This gives the inequality in the proposition

$$
U(x, y) \geq|h|+\frac{\omega^{2}}{2 r^{2}} .
$$

Conversely, if this inequality holds, it must be shown that there exists $(u, w) \in \mathbf{R}^{6}$ for which the point $(x, y, u, w)$ satisfies $H=h$ and $|\Omega|=\omega$. Let $v \in \mathbf{R}$ satisfy $\frac{1}{2} v^{2}=U(x, y)-|h|-\frac{\omega^{2}}{2 r^{2}}$ and let $e \in \mathbf{R}^{3}$ be any unit vector which is orthogonal to both $x$ and $y$. Then set

$$
u=\frac{v}{r} x+\frac{\omega}{r^{2}}(e \times x), \quad w=\frac{v}{r} y+\frac{\omega}{r^{2}}(e \times y) .
$$

Since $x$ and $e \times x$ are orthogonal, $|u|^{2}=\left(v^{2}+\frac{\omega^{2}}{r^{2}}\right) \frac{|x|^{2}}{r^{2}}$. Similarly $|w|^{2}=$ $\left(v^{2}+\frac{\omega^{2}}{r^{2}}\right) \frac{|y|^{2}}{r^{2}}$. Therefore, by the choice of $v$, the energy is

$$
H(x, y, u, w)=\frac{1}{2} v^{2}+\frac{\omega^{2}}{2 r^{2}}-U(x, y)=-|h|=h .
$$

Also the angular momentum is

$$
\Omega=\mu_{1} x \times u+\mu_{2} y \times w=\frac{\omega}{r^{2}}\left(\mu_{1} x \times(e \times x)+\mu_{2} y \times(e \times y)\right)=\omega e .
$$

Therefore $|\Omega|=\omega$ as required.

It is clear from the definitions that $\mathcal{H}_{1}(h, \omega)$ is just the projection of $\mathcal{H}(h, \omega)$ onto $\Sigma$, i.e., $(\xi, \eta) \in \mathcal{H}_{1}(h, \omega)$ if and only if there exists $r>0$ such that $(x, y)=$ $(r \xi, r \eta) \in \mathcal{H}(h, \omega)$. From the first part of the proof, $(r \xi, r \eta) \in \mathcal{H}(h, \omega)$ if and only if

$$
\frac{1}{r} U(\xi, \eta) \geq|h|+\frac{\omega^{2}}{2 r^{2}} .
$$

Thus $(\xi, \eta) \in \mathcal{H}_{1}(h, \omega)$ if and only if the quadratic equation

$$
2|h| r^{2}-2 U(\xi, \eta) r+\omega^{2}=0
$$

has a positive root and this reduces to the condition $U(\xi, \eta) \geq \lambda$.

The quantity $\lambda$ is clearly an integral of the three-body problem. Although the three-body problem has two natural integrals $h, \omega$, the flows on integral manifolds with the same values of $\lambda$ are equivalent by rescaling the variables. So there is essentially only one parameter.

The three-body problem has a rotational symmetry which was eliminated from the restricted problem when the coordinate axes were chosen. For the spatial problem, the three-dimensional rotation group $\mathrm{SO}(3)$ acts freely on each of the integral manifolds $\mathcal{M}(h, \omega)$. Thus the three-body flow projects to flows on the reduced integral manifolds

$$
\tilde{\mathcal{M}}(h, \omega)=\mathcal{M}(h, \omega) / \mathrm{SO}(3) .
$$

One can also defined reduced Hill's regions and reduced normalized Hill's regions

$$
\tilde{\mathcal{H}}(h, \omega)=\mathcal{H}(h, \omega) / \mathrm{SO}(3), \quad \tilde{\mathcal{H}}_{1}(h, \omega)=\mathcal{H}_{1}(h, \omega) / \mathrm{SO}(3) .
$$


However, the action of the group on the configuration space is not free and the quotient spaces are manifolds-with-boundary. As will be shown below, the $\tilde{\mathcal{H}}_{1}(h, \omega)$ can be visualized in two dimensions, much like the Hill's regions for the planar restricted problem.

Before discussing this, consider the planar problem. The integral manifolds and Hill's regions are obtained by simply setting the third coordinates of the vectors $x, y, u, w$ to zero. The most natural symmetry group is the two-dimensional rotation group $\mathrm{SO}(2)$, and one can define reduced manifolds as above. However, these are not just the obvious subsets of the reduced manifolds of the spatial problem, because the action of $\mathrm{SO}(3)$ on the planar points includes rotations by $\pi$ around axes in the plane. When viewed as maps of the plane, these are reflections, not rotations. The planar part of the reduced spatial manifolds are quotient spaces under the action of $\mathrm{O}(2)$ rather than $\mathrm{SO}(2)$.

The best way to visualize the Hill's regions is to use a variant of the Hopf map. First consider the planar problem, so that $x, y \in \mathbf{R}^{2}$. Then define $h: \mathbf{R}^{2} \times \mathbf{R}^{2} \rightarrow \mathbf{R}^{3}$ by $h(x, y)=\left(h_{1}, h_{2}, h_{3}\right)$, where

$$
\begin{aligned}
& h_{1}(x, y)=\mu_{1}|x|^{2}-\mu_{2}|y|^{2}, \\
& h_{2}(x, y)=2 \sqrt{\mu_{1} \mu_{2}} x \cdot y \\
& h_{3}(x, y)=2 \sqrt{\mu_{1} \mu_{2}}\left(x_{1} y_{2}-x_{2} y_{1}\right) .
\end{aligned}
$$

With $\mu_{1}=\mu_{2}=1$ this is the standard Hopf map which restricts to the Hopf fibration taking the unit three-sphere onto the unit two-sphere. For general $\mu_{i}, h$ takes the normalized configuration space for the planar three-body problem onto the unit two-sphere. For the spatial case, define a map $h: \mathbf{R}^{3} \times \mathbf{R}^{3} \rightarrow \mathbf{R}^{5}$ by replacing the third component by the cross-product

$$
\begin{aligned}
& h_{1}(x, y)=\mu_{1}|x|^{2}-\mu_{2}|y|^{2}, \\
& h_{2}(x, y)=2 \sqrt{\mu_{1} \mu_{2}} x \cdot y, \\
& h_{3}(x, y)=2 \sqrt{\mu_{1} \mu_{2}} x \times y \in \mathbf{R}^{3} .
\end{aligned}
$$

To unify notation for the planar and spatial cases, define the cross product to be scalar $x \times y=x_{1} y_{2}-x_{2} y_{1} \in \mathbf{R}$ when $x, y \in \mathbf{R}^{2}$.

One can check that $h$ satisfies

$$
h_{1}^{2}+h_{2}^{2}+\left|h_{3}\right|^{2}=\left(\mu_{1}|x|^{2}+\mu_{2}|y|^{2}\right)^{2}=r^{4} .
$$

In the planar case this shows that $h$ restricts to a map $h: \Sigma \rightarrow \mathbf{S}^{2}$ taking the normalized configuration space onto the unit two-sphere. In the spatial case $h: \Sigma \rightarrow$ $\mathbf{S}^{4}$, but one can define a map $\bar{h}: \Sigma \rightarrow \mathbf{S}^{2}$ by $\bar{h}(x, y)=\left(h_{1}(x, y), h_{2}(x, y),\left|h_{3}(x, y)\right|\right)$ which maps $\Sigma$ onto the upper hemisphere of the unit two-sphere.

The fibers of these maps are just the orbits of the symmetry groups acting on $\Sigma$. Thus the points of the two-sphere or its upper hemisphere represent the possible shapes of the triangle formed by the three masses without regard to size or orientation. For this reason, $S^{2}$ will be called the shape sphere of the three-body problem. Note that the collinear shapes are characterized by $h_{3}(x, y)=0$ in both the planar and spatial cases. Thus the collinear configurations form the equator of the shape sphere.

Since the restricted potential function $U: \Sigma \rightarrow \mathbf{R}$ is invariant under the action of the symmetry groups, it induces a well-defined function $V: \mathbf{S}^{2} \rightarrow \mathbf{R}$ (in 
the spatial case, extend symmetrically to the lower hemisphere). Let $(\rho, \theta, \phi)$ denote the usual spherical coordinates in $\mathbf{R}^{3}$. Composing with $\bar{h}$ gives functions $(\rho(x, y), \tau(x, y), \phi(x, y))$, where

$$
\rho^{2}=\left(\mu_{1}|x|^{2}+\mu_{2}|y|^{2}\right)^{2}=r^{4}
$$

and

$$
h_{1}(x, y)=\rho \cos \theta \cos \phi, \quad h_{2}(x, y)=\rho \sin \theta \cos \phi, \quad\left|h_{3}(x, y)\right|=\rho \sin \phi .
$$

It is possible to work out a complicated explicit formula for $V(\tau, \phi)$, but it is easier to work with Cartesian or Jacobi coordinates when computations are necessary.

Figure 5 shows some of the level curves of $V$ using stereographic projection. The pole of the projection is chosen to be the double collision configuration with $q_{1}=q_{2}$. This is a collinear configuration so it lies on the equator of the shape sphere. The analogy with the planar restricted problem is clear. It follows from Proposition 3.1 that the reduced normalized Hill's regions, $\mathcal{H}_{1}(h, \omega)$, are given by $V \geq \lambda$ (shaded in the figure).

3.3. The collinear relative equilibria. In this section, the analogue of the equilibrium point $L_{2}$ will be described. A relative equilibrium solution of the threebody problem is a solution which becomes an equilibrium for the quotient flow on $\tilde{\mathcal{M}}(h, \omega)$. It can be shown (with some effort) that a relative equilibrium solution must rotate rigidly at constant speed around an axis orthogonal to the plane of containing the three masses [3], [14]. In particular, if one chooses the rotation axis vertical, it will be a solution of the planar problem.

Let $\alpha \in \mathbf{R}^{3}$ denote the constant angular velocity vector of such a uniformly rotating solution. Then $\alpha \cdot x=\alpha \cdot y=0$ and the velocities are given by $u=$ $\alpha \times x, w=\alpha \times y$. It follows that $\dot{u}=\alpha \times(\alpha \times x)=(a \cdot x) a-(a \cdot a) x=-|\alpha|^{2} x$. Similarly $\dot{w}=-|\alpha|^{2} y$. From the equations of motions (3.4), the configuration must satisfy

$$
U_{x}+\mu_{1}|\alpha|^{2} x=U_{y}+\mu_{2}|\alpha|^{2} y=0 .
$$

Conversely, given such a configuration, any choice of a vector $\alpha$ orthogonal to $x$ and $y$ yields a relative equilibrium solution. As in the restricted problem, the relative equilibria arise from critical points of the Newtonian potential.

Proposition 3.2. A configuration $(x, y) \in \mathbf{R}^{2 d}$ satisfies (3.10) for some constant $|\alpha|^{2}$ if and only if the corresponding normalized configuration $(\xi, \eta) \in \Sigma$ is a critical point of the restriction of the Newtonian potential to $\Sigma, U(\xi, \eta)$. Moreover, for the corresponding periodic solution, the following equations hold:

$$
|\alpha|^{2}=U(\xi, \eta) r^{-3}, \quad h=-\frac{1}{2} U(\xi, \eta) r^{-1}, \quad \omega^{2}=U(\xi, \eta) r .
$$

Proof. By homogeneity of $U,(x, y)$ satisfies (3.10) with constant $|\alpha|^{2}$ if and only if $(\xi, \eta)$ satisfies the same equation with constant $|\alpha|^{2} r^{-1}$. Since $\Sigma$ is defined by the constraint $g(\xi, \eta)=\mu_{1}|\xi|^{2}+\mu_{2}|\eta|^{2}=1$ the critical points of the restricted potential satisfy

$$
\nabla U(\xi, \eta)+l \nabla g(\xi, \eta)=0
$$

for some Lagrange multiplier, $l$. Taking dot products with $(\xi, \eta)$ gives $-U(\xi, \eta)+$ $2 l=0$ and it follows that $2 l=U(\xi, \eta)>0$. Setting $2 l=|\alpha|^{2} r^{3}$ gives (3.10) and the formula for $|\alpha|^{2}$. The other two formulas follow by simple computations. 

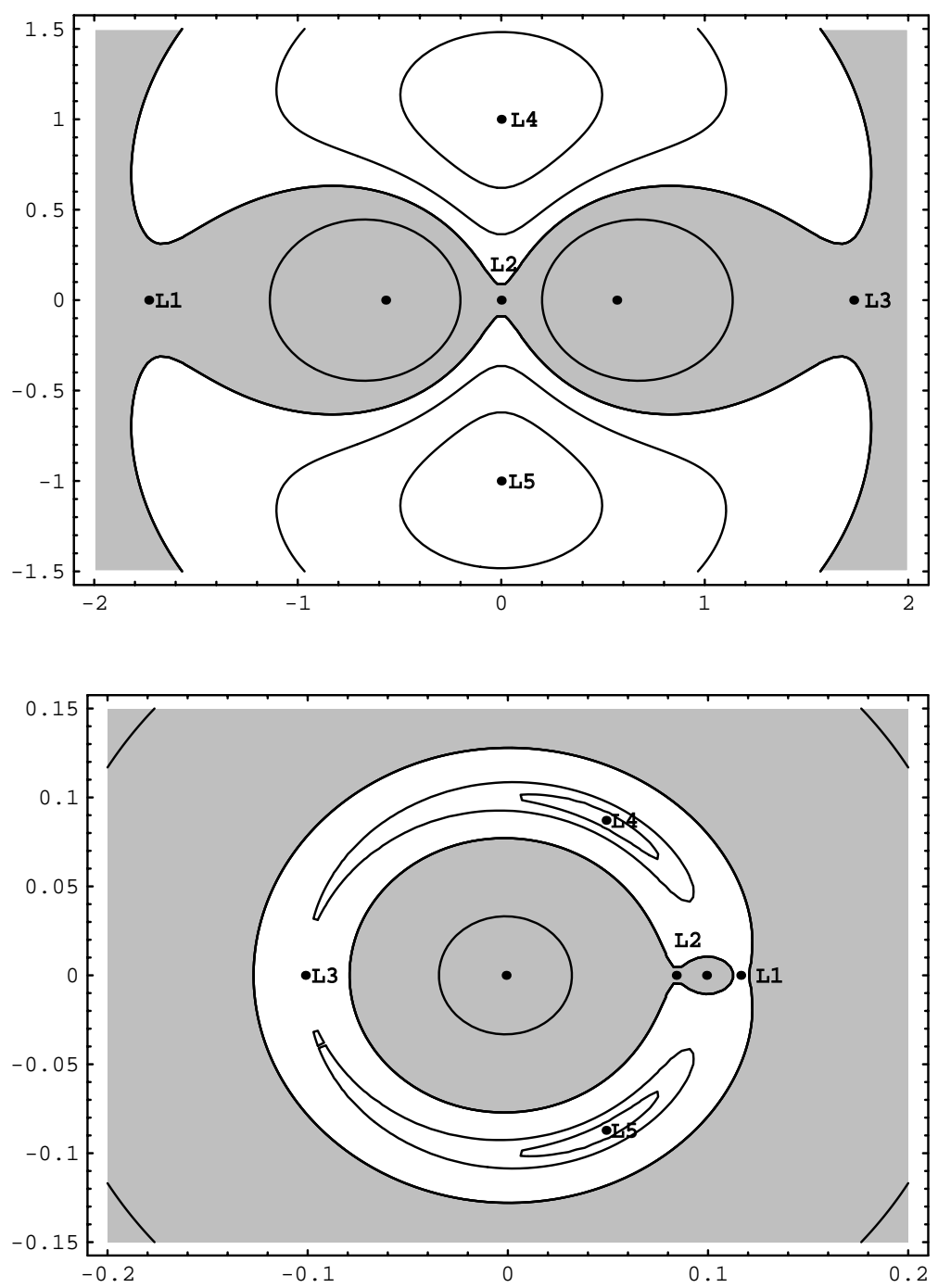

FiguRE 5. Hill's regions and Lagrange points for the unrestricted three-body problems with $m_{1}=m_{2}=m_{3}=1$ (top) and $m_{1}=$ $81, m_{2}=1, m_{3}=0.01$ (bottom).

Note that the integral $\lambda=\sqrt{2|h| \omega^{2}}=\sqrt{2|h||\Omega|^{2}}$ equals $U(\xi, \eta)$. Thus $\lambda$ is just the critical value of the corresponding critical point in $\Sigma$.

Because of the symmetry, finding critical points of $\left.U\right|_{\Sigma}$ is equivalent to finding critical points of the induced function $V: \mathbf{S}^{2} \rightarrow \mathbf{R}$. It turns out that there are always exactly five such critical points just as in the restricted problem [14]. Two of them are the equilateral triangles, labelled as $L_{4}, L_{5}$ in Figure 4 . The other three are collinear and so appear on the equator of the shape sphere or on the horizontal axis in the stereographic projection. 
Without loss of generality, one can focus attention on the collinear configurations for which $m_{3}$ lies between $m_{1}$ and $m_{2}$. The polar angle $\theta$ in spherical coordinates provides a natural coordinate for the collinear configurations. Alternatively, one can use the distance ratio $\rho=r_{13} / r_{12}$. Some computation shows that at the relative equilibrium the following famous fifth degree equation due to Euler must hold [14]:

$$
\begin{aligned}
\left(m_{1}+m_{2}\right) \rho^{5} & -\left(2 m_{1}+3 m_{2}\right) \rho^{4}+\left(m_{1}+3 m_{2}+2 m_{3}\right) \rho^{3} \\
& -\left(m_{1}+3 m_{3}\right) \rho^{2}+\left(2 m_{1}+3 m_{3}\right) \rho-\left(m_{1}+m_{3}\right)=0 .
\end{aligned}
$$

It is not hard to see that it has a unique root $\bar{\rho} \in(0,1)$. Note that $\rho=0,1$ correspond to binary collision configurations between masses $m_{1}, m_{3}$ or $m_{2}, m_{3}$. If $\theta_{13}, \theta_{23}$ are the theta coordinates of these collision configurations, then there is a unique critical point $\bar{\theta}$ of the collinear potential $V(\tau, 0)$ on the interval $\left(\theta_{13}, \theta_{23}\right)$.

Just as for the restricted problem, a local calculation at the critical point can be used to prove the existence of a normally hyperbolic sphere nearby. Using Cartesian coordinates in the spatial three-body problem, the phase space has dimension 18, the integral manifolds $\mathcal{M}(h, \omega)$ have dimension 10 and the reduced integral manifolds $\tilde{\mathcal{M}}(h, \omega)$ have dimension 7 . In an appropriate basis, the $18 \times 18$ matrix of the linearized differential equations at the collinear relative equilibrium splits into three $4 \times 4$ blocks and three $2 \times 2$ of the form

$$
A_{i}=\left[\begin{array}{cccc}
0 & 0 & 1 & 0 \\
0 & 0 & 0 & 1 \\
\bar{\lambda}+2 d_{i} & 0 & 0 & -2 \\
0 & \bar{\lambda}-d_{i} & 2 & 0
\end{array}\right], \quad B_{i}=\left[\begin{array}{cc}
0 & 1 \\
\bar{\lambda}-d_{i} & 0
\end{array}\right], \quad i=1,2,3 .
$$

Note the similarity with (2.3).

Here $d_{i}$ are the eigenvalues of a certain $3 \times 3$ matrix denoted $M^{-1} K$ below (see (3.27) $)$. As described below, the eigenvalues are $d_{1}=0, d_{2}=\bar{\lambda}$ and another eigenvalue, $d_{3}$. The characteristic polynomials are

$$
P_{A_{i}}(\zeta)=\zeta^{2}+\left(2 \bar{\lambda}-d_{i}\right) \zeta-\left(d_{i}-\bar{\lambda}\right)\left(2 d_{i}+\bar{\lambda}\right), \quad P_{B}(\zeta)=\zeta+d_{i},
$$

where $\zeta$ represents the square of an eigenvalue.

The eigenvalue $d_{1}=0$ is associated to the translational symmetry of the threebody problem. The corresponding solutions of (3.14) are $\zeta=-\bar{\lambda},-\bar{\lambda}, 0$ yielding eigenvalues $\pm i \sqrt{\lambda}, \pm i \sqrt{\lambda}, 0,0$. However, by working in a reduced phase space, for example by using Jacobi coordinates, one can ignore these six eigenvalues. The eigenvalue $d_{2}=\bar{\lambda}$ is associated to the rotational and scaling symmetry of the problem. The corresponding solutions of (3.14) are also $\zeta=-\bar{\lambda},-\bar{\lambda}, 0$. By fixing $|\Omega|$ (but not $h$ ) and quotienting out the rotational symmetry one can reduce to an eight-dimensional space. Two of the eight eigenvalues are $\pm i \sqrt{\bar{\lambda}}$ coming from $A_{2}$. The other six eigenvalues come from $A_{3}, B_{3}$. It turns out that $d_{3}>\bar{\lambda}$. Just as in the restricted problem this leads to two real eigenvalues $\pm \alpha$ and two imaginary eigenvalues $\pm i \beta$ for $A_{3}$ and eigenvalues $\pm i \sqrt{d_{3}}$ for $B_{3}$. Thus, in the eight-dimensional reduced space, the restpoint corresponding to the collinear relative equilibrium has two real and six imaginary eigenvalues. Moreover, further computations involving the eigenvectors shows that the Hessian of the energy function is positive definite on the six-dimensional center subspace. Thus by intersecting the nearby reduced energy manifolds with the center manifold of the restpoint one finds normally hyperbolic invariant five-dimensional spheres. In the planar case there is one less 
imaginary pair and so there are invariant three-spheres. As in the restricted problem, it is not clear what happens to these spheres as one moves to integral manifolds far from the relative equilibrium. This is part of the motivation for the construction of isolating blocks in the next section.

3.4. The isolating block. It is natural to think of the angle $\theta$ on $\mathbf{S}^{2}$ as analogous to the variable $x$ in the restricted problem. This suggests attempting to construct an isolating block of the form $a \leq \theta \leq b$, where $[a, b]$ is an interval such that $\theta_{13}<a<\bar{\theta}<b<\theta_{23}$. For this to be isolating one needs to have $\ddot{\theta}>0$ whenever $\theta=b, \dot{\theta}=0$ and $\ddot{\theta}<0$ whenever $\theta=a, \dot{\theta}=0$. Now from (3.9),

$$
\begin{aligned}
\dot{\theta} & =\frac{h_{1} \dot{h}_{2}-h_{2} \dot{h}_{1}}{h_{1}^{2}+h_{2}^{2}}, \\
\left.\ddot{\theta}\right|_{\dot{\theta}=0} & =\frac{h_{1} \ddot{h}_{2}-h_{2} \ddot{h}_{1}}{h_{1}^{2}+h_{2}^{2}} .
\end{aligned}
$$

To facilitate the computations, introduce several bilinear functions. Let $(x, y)$, $(u, w)$ be two vectors in $\mathbf{R}^{2 d}$ and define

$$
\begin{aligned}
& b_{0}(x, y ; u, w)=\mu_{1} x \cdot u+\mu_{2} y \cdot w, \\
& b_{1}(x, y ; u, w)=\mu_{1} x \cdot u-\mu_{2} y \cdot w, \\
& b_{2}(x, y ; u, w)=\sqrt{\mu_{1} \mu_{2}}(x \cdot w+y \cdot u), \\
& b_{3}(x, y ; u, w)=\sqrt{\mu_{1} \mu_{2}}(x \times w-y \times u), \\
& \Omega(x, y ; u, w)=\mu_{1} x \times u+\mu_{2} y \times w .
\end{aligned}
$$

Note that the components of the Hopf map $h$ in the last section are obtained by setting $(u, w)=(x, y)$, i.e., $h_{i}(x, y)=b_{i}(x, y ; x, y), i=1,2,3$. Also $h_{0}(x, y)=$ $b_{0}(x, y ; x, y)=r^{2}$ is the moment of inertia. It is useful to introduce the notation $k_{i}(u, w)=b_{i}(u, w ; u, w), i=0,1,2,3$. Note, in particular, that $k_{0}(u, w)=\mu_{1}|u|^{2}+$ $\mu_{2}|w|^{2}=2 T$, twice the kinetic energy.

These functions satisfy the identity

$$
h_{0} k_{0}=b_{1}^{2}+b_{2}^{2}+\left|b_{3}\right|^{2}+|\Omega|^{2} .
$$

This can be verified by straightforward computation using the vector identity

$$
(a \times b) \cdot(c \times d)=(a \cdot b)(c \cdot d)-(a \cdot d)(b \cdot c) .
$$

Setting $(u, w)=(x, y)$ gives the Hopf identity (B.8). One can also check, for example by differentiating (3.8), that

$$
h_{0} b_{0}=h_{1} b_{1}+h_{2} b_{2}+h_{3} \cdot b_{3} .
$$

Indeed, assuming that $(u, w)=(\dot{x}, \dot{y})$, one has

$$
\dot{h}_{i}=2 b_{i}, \quad i=0,1,2,3 .
$$

Note also that the assumption that $\dot{\theta}=0$ is equivalent to $h_{1} b_{2}-h_{2} b_{1}=0$.

Now from equations (3.4) and (3.7) a short computation shows that

$$
\frac{1}{2}\left(h_{1} \ddot{h}_{2}-h_{2} \ddot{h}_{1}\right)=\tilde{D} U(x, y)+G(x, y, u, v),
$$

where

$$
\begin{aligned}
\tilde{D} U & =h_{1}\left(\sqrt{\frac{\mu_{1}}{\mu_{2}}} x \cdot U_{y}+\sqrt{\frac{\mu_{2}}{\mu_{1}}} y \cdot U_{x}\right)-h_{2}\left(x \cdot U_{x}-y \cdot U_{y}\right), \\
G & =h_{1}\left(2 \sqrt{\mu_{1} \mu_{2}} u \cdot w\right)-h_{2}\left(\mu_{1}|u|^{2}-\mu_{2}|w|^{2}\right)=h_{1} k_{2}-h_{2} k_{1} .
\end{aligned}
$$


To determine the sign of $\ddot{\theta}$ one needs to understand the relative sizes of the $\tilde{D} U$ and $G$. The next result provides a simple interpretation of the rather complicated looking derivative $\tilde{D} U$ in terms of the induced potential $V$ on $\mathbf{S}^{2}$.

Lemma 3.3. For $(x, y) \in \mathbf{R}^{6}$, let $(\xi, \eta) \in \Sigma$ be the corresponding normalized configuration and $(\rho(x, y), \theta(x, y), \phi(x, y))$ the spherical coordinates (3.9). Then

$$
\tilde{D} U(x, y)=r \tilde{D} U(\xi, \eta)=2 r V_{\theta}(\theta, \phi) .
$$

Proof. The first formula follows from definition of $\tilde{D} U$ and the homogeneity of the functions $U(x, y)$ and $h_{i}(x, y)$. The rotational symmetry of the potential and the fact that the map $\bar{h}$ is the quotient map for the symmetry group implies that $U(x, y)$ induces a function $F$ on $\mathbf{R}^{3}$ such that

$$
U(x, y)=F\left(h_{1}(x, y), h_{2}(x, y),\left|h_{3}(x, y)\right|\right) .
$$

Since $V$ is also induced by $U$, it follows that $V=\left.F\right|_{\mathbf{S}^{2}}$.

For configurations with $x \times y \neq 0$, the chain rule gives

$$
\begin{aligned}
& U_{x}=2 \mu_{1} x F_{1}+2 \sqrt{\mu_{1} \mu_{2}} y F_{2}+2 \sqrt{\mu_{1} \mu_{2}} \frac{y \times(x \times y)}{|x \times y|} F_{3}, \\
& U_{y}=-2 \mu_{1} y F_{1}+2 \sqrt{\mu_{1} \mu_{2}} x F_{2}-2 \sqrt{\mu_{1} \mu_{2}} \frac{x \times(x \times y)}{|x \times y|} F_{3},
\end{aligned}
$$

where $F_{i}$ are the partial derivatives of $F$. Substituting these into the definition of $\tilde{D} U$ and simplifying gives

$$
\tilde{D} U(x, y)=2 r^{2}\left(h_{1} F_{2}-h_{2} F_{1}\right), \quad \tilde{D} U(\xi, \eta)=2\left(h_{1} F_{2}-h_{2} F_{1}\right) .
$$

Since all the functions occurring here are continuous on the set of (nonsingular) configurations with $x \times y=0$, the formulas remain valid there.

On the other hand, since $V(\theta, \phi)=F(\cos \theta \cos \phi, \sin \theta \cos \phi, \sin \phi)$,

$$
V_{\theta}(\theta, \phi)=-\sin \theta \cos \phi F_{1}+\cos \theta \cos \phi F_{2} .
$$

If $(\theta, \phi)$ are the spherical coordinates of a normalized configurations $(\xi, \eta)$, (3.9) gives

$$
V_{\theta}(\theta, \phi)=-h_{2} F_{1}+h_{1} F_{2}
$$

which together with (3.19) proves the second formula of the lemma.

The next result is crucial because it provides an estimate for $G(x, y, u, w)$ depending only on the configuration variables.

Lemma 3.4. Let $(x, y, u, w) \in \mathcal{M}(h, \omega)$ and suppose that $h_{1} b_{2}-h_{2} b_{1}=0$. Then

$$
|G(x, y, u, w)|^{2} \leq 4 r^{2}\left(U(\xi, \eta)^{2}-\lambda^{2}\right),
$$

where $\lambda=\sqrt{2|h| \omega^{2}}$.

Proof. Begin by splitting the velocity vector $(u, w)$ into components tangent and normal to the ellipsoid $\mu_{1}|x|^{2}+\mu_{2}|y|^{2}=r^{2}$ containing $(x, y)$. Define $v \in \mathbf{R}$ and $\hat{u}, \hat{w} \in \mathbf{R}^{2 d}$ by

$$
v=\frac{1}{r^{2}}\left(\mu_{1} x \cdot u+\mu_{2} y \cdot w\right), \quad u=v x+\hat{u}, \quad w=v y+\hat{w} .
$$

Then

$$
\mu_{1} x \cdot \hat{u}+\mu_{2} y \cdot \hat{w}=0
$$


which is just the condition that $(\hat{u}, \hat{w})$ be tangent to the ellipsoid. Using the bilinearity of the functions $b_{i}$, it follows that

$$
b_{i}(x, y ; u, w)=v h_{i}+\hat{b}_{i}, \quad k_{i}(u, w)=v^{2} h_{i}+2 v \hat{b}_{i}+\hat{k}_{i}, \quad i=0,1,2,3,
$$

where $\hat{b}_{i}=b_{i}(x, y ; \hat{u}, \hat{w})$ and $\hat{k}_{i}=k_{i}(\hat{u}, \hat{w})$. Substituting these into the formulas above shows that

$$
h_{1} b_{2}-h_{2} b_{1}=h_{1} \hat{b}_{2}-h_{2} \hat{b}_{1}=0
$$

and

$$
G=h_{1} k_{2}-h_{2} k_{1}=2 v\left(h_{1} \hat{b}_{2}-h_{2} \hat{b}_{1}\right)+h_{1} \hat{k}_{2}-h_{2} \hat{k}_{1}=h_{1} \hat{k}_{2}-h_{2} \hat{k}_{1}=\hat{G} .
$$

Also (3.17) gives

$$
h_{1} \hat{b}_{1}+h_{2} \hat{b}_{2}+h_{3} \cdot \hat{b}_{3}=0
$$

while (3.16) becomes

$$
h_{0} k_{0}=r^{2}(2 T)=v^{2}+\hat{b}_{1}^{2}+\hat{b}_{2}^{2}+\left|\hat{b}_{3}\right|^{2}+|\Omega|^{2} .
$$

Now it turns out that there is a very simple formula for $\hat{G}$, namely,

$$
\hat{G}=h_{1} \hat{k}_{2}-h_{2} \hat{k}_{1}=2 \hat{b}_{3} \cdot \Omega \text {. }
$$

To see this, note that $\Omega(x, y ; u, w)=\Omega(x, y ; \hat{u}, \hat{w})$ and so

$$
2 \hat{b}_{3} \cdot \Omega=2 \sqrt{\mu_{1} \mu_{2}}(x \times \hat{w}-y \times \hat{u}) \cdot\left(\mu_{1} x \times \hat{u}+\mu_{2} y \times \hat{w}\right) .
$$

Multiplying out the dot product and simplifying gives

$$
2 \hat{b}_{3} \cdot \Omega=h_{1} \hat{k}_{2}-h_{2} \hat{k}_{1}+(y \cdot \hat{u}-x \cdot \hat{w})\left(\mu_{1} x \cdot \hat{u}+\mu_{2} y \cdot \hat{w}\right),
$$

and the last term vanishes by (3.20).

To get the estimate in the lemma, note that $|G|^{2}=|\hat{G}|^{2} \leq 4\left|\hat{b}_{3}\right|^{2}|\Omega|^{2}$. From (3.21) and the energy equation, the vectors $\hat{b}_{3}, \Omega$ satisfy $\left|\hat{b}_{3}\right|^{2}+|\Omega|^{2} \leq r^{2}(2 U+2 h)$ which can be written in the form

$$
\alpha^{2}+\beta^{2}+\frac{\lambda^{2}}{\beta^{2}} \leq 2 U(\xi, \eta)
$$

where $\alpha, \beta \in \mathbf{R}^{+}$satisfy

$$
\alpha^{2}=\frac{\left|\hat{b}_{3}\right|^{2}}{r}, \quad \beta^{2}=\frac{|\Omega|^{2}}{r} .
$$

Maximizing $\alpha^{2} \beta^{2}$ subject to the constraint (3.22) gives $\alpha^{2} \beta^{2} \leq U^{2}-\lambda^{2}$. This gives $|\hat{G}|^{2} \leq 4 r^{2}\left(U^{2}-\lambda^{2}\right)$ as required.

Combining these two lemmas gives convenient sufficient conditions for the existence of the isolating block.

Theorem 3.5. Let $h\langle 0, \omega\rangle 0$ be chosen so that the Hill's region $\mathcal{H}(h, \omega)$ is contained in the domain of $\theta(x, y)$. Define $B_{(h, \omega)}(a, b) \subset \mathcal{M}(h, \omega)$ by the inequality $a \leq \theta(x, y) \leq b$, where $\theta_{13}<a<\theta_{c}<b<\theta_{23}$. Then $B_{(h, \omega)}(a, b)$ is an isolating block for the flow on $\mathcal{M}(h, \omega)$ provided that

$$
\tilde{D} U^{2}>4\left(U^{2}-\lambda^{2}\right)
$$

for all $(\xi, \eta) \in \mathcal{H}_{1}(h, \omega)$ such that $\theta(\xi, \eta)=a$ or $\theta(\xi, \eta)=b$, where $\lambda=\sqrt{2|h| \omega^{2}}$. Equivalently, it suffices to show that, for all $(\theta, \phi) \in \tilde{\mathcal{H}}_{1}(h, \omega)$ with $\theta=a, b$,

$$
V_{\theta}^{2}>V^{2}-\lambda^{2} \text {. }
$$


Proof. The equivalence of these two conditions follows from the second formula of Lemma 3.3. It will be shown that $\left.\ddot{\theta}\right|_{\dot{\theta}=0}>0$ on the set $S_{b}=\{(x, y, u, w) \in$ $\mathcal{M}(h, \omega): \theta(x, y)=b\}$. The proof that $\left.\ddot{\theta}\right|_{\dot{\theta}=0}<0$ when $\theta=a$ is similar.

By the computations at the beginning of this section, the sign of $\left.\ddot{\theta}\right|_{\dot{\theta}=0}>0$ is the same as that of $\tilde{D} U(x, y)+G(x, y, u, w)$. By Lemma 3.3, $\tilde{D} U(x, y)$ has the same sign as $V_{\theta}(\theta, \phi)$. The fact that $\bar{\theta}$ is the only critical point of the collinear potential $V(\theta, 0)$ on $\left(\theta_{13}, \theta_{23}\right)$ shows that $V_{\theta}(b, 0)>0$. Thus $\tilde{D} U(x, y)>0$ at any collinear configuration in $S_{b}$. Since $S_{b}$ is a connected set, it suffices to show that $(\tilde{D} U)^{2}>|G|^{2}$ holds. Then $\tilde{D} U>0$ everywhere in $S_{b}$ and also

$$
\tilde{D} U+G \geq \tilde{D} U-|G|>0 .
$$

Finally, the lemmas show that the condition $(\tilde{D} U)^{2}>|G|^{2}$ is equivalent to the ones given in the theorem.

The second condition in Theorem 3.5 reduces the whole question to the verification of an inequality on a one-dimensional set, namely the $\operatorname{arcs}$ in $\tilde{\mathcal{H}}_{1} \subset \mathbf{S}^{2}$ with $\theta=a, b$. This is similar to the inequality (2.4) for $x=a, b$ in the planar restricted problem. However, the verification of the required inequality is still very complicated. Whether or not the inequality holds depends on the parameters $\lambda, a, b$ as well as on the masses, $m_{i}$. It is possible to use rigorous computer calculations to verify the inequality in certain cases (see section 3.5). But to give some indication of what is involved, some nonrigorous numerical calculation will be described now.

For fixed masses, $m_{i}$, and fixed $\lambda$, define a function on the shape sphere by

$$
W(\theta, \phi)=V_{\theta}^{2}(\theta, \phi)-V^{2}(\theta, \phi)+\lambda^{2} .
$$

Then the isolating block condition is that $W>0$ on the part of the $\operatorname{arcs} \theta=a, b$ in the Hill's region. Figures 6 , 7 show stereographic plots of the shape sphere for several choices of $m_{i}$ and $\lambda$. On each plot, the forbidden region $W \leq 0$ shaded and several $\operatorname{arcs}$ of the form $\theta=c$ are shown. If the $\operatorname{arc} \theta=c$ arc lies entirely in the unshaded region, then $c$ is an admissible boundary value for an isolating block.

Figure 6] shows the equal-mass and (not quite) lunar problems, respectively. For each problem there are three plots with different values of the parameter $\lambda$. The top value is chosen close to the critical value $\bar{\lambda}$ and the others have lower values. In both cases the result is the same. For $\bar{\lambda}-\lambda$ small, the forbidden region has several components, one near the Lagrange point and the others far away. Between them, there is a large region with $W>0$ and many choices of the $\operatorname{arc} \theta=c$ satisfy the isolating block inequality. One can construct an infinitesimal block with boundaries very close to the Lagrange point or much larger blocks. As the energy and angular momentum parameter, $\lambda$, is decreased, the forbidden region grows, making it harder to choose $c$. One can make the block neither too small nor too large. Finally, decreasing $\lambda$ further causes the forbidden regions to join and it is no longer possible to choose any arc satisfying the isolating block inequality. Figure 7 shows the behavior when the middle mass is taken substantially larger than the others. In this case, even infinitesimal blocks are impossible. Apparently isolating blocks of the form considered here do not exist for any choice of $\lambda$.

If the masses are fixed, one can ask for the values of the parameters $(c, \lambda)$ for which the isolating block inequality holds. The picture showing these parameters 

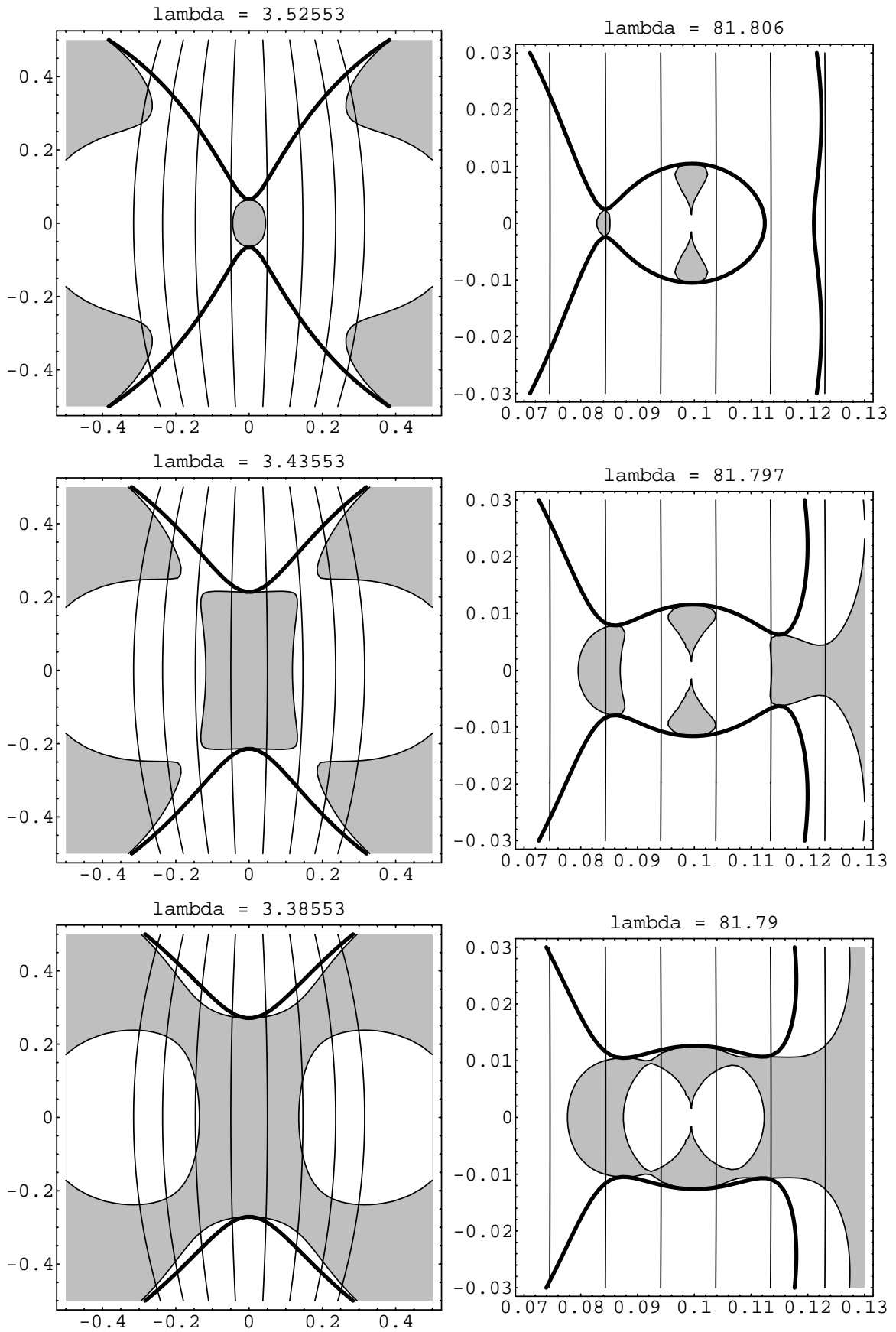

FIGURE 6. Stereographic projection of the shape sphere showing forbidden regions $W \leq 0$ (shaded) and $\operatorname{arcs} \theta=c$. The dark curves show the boundaries of the Hill's regions. The left column shows the equal mass case $m_{1}=m_{2}=m_{3}=1, \bar{\lambda}=3.53553$ for three choices of $\lambda<\bar{\lambda}$. The right column shows $m_{1}=81, m_{2}=1, m_{3}=$ $0.01, \bar{\lambda}=81.807$. 

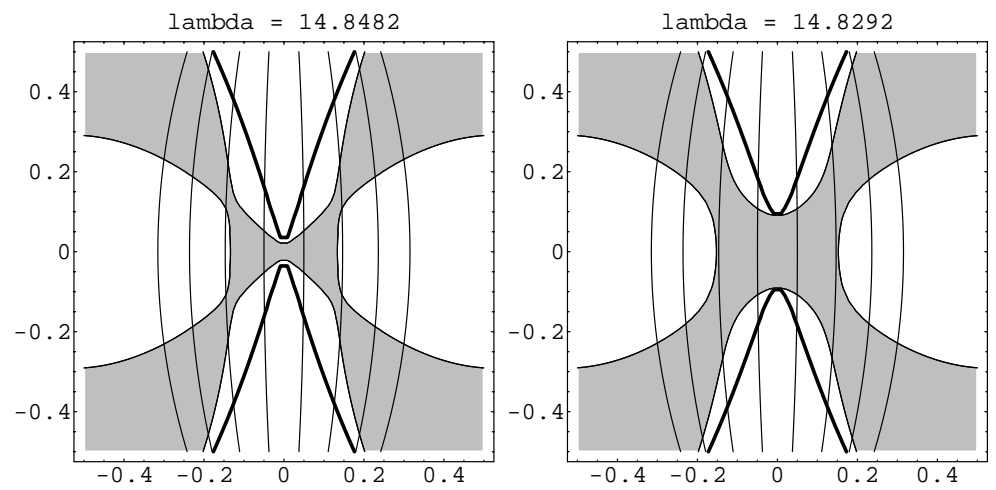

FIGURE 7. Stereographic projection of the shape sphere showing forbidden regions $W \leq 0$ (shaded) and Arcs $\theta=c$ for $m_{1}=m_{2}=$ $1, m_{3}=5, \bar{\lambda}=14.8492$. This time it is impossible to find an arc avoiding the forbidden region.
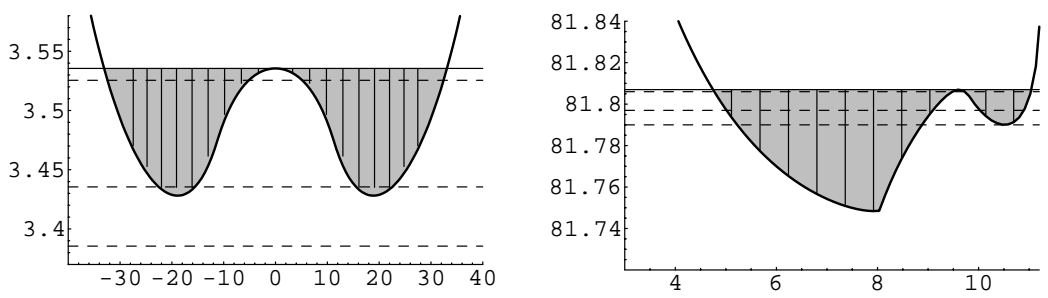

FiguRE 8. Isolating block bifurcation diagrams for $m_{1}=m_{2}=$ $m_{3}=1$ (left) and $m_{1}=81, m_{2}=1, m_{3}=0.01$ (right). The horizontal axis shows angles $\theta=c$ in degrees, and the vertical axis is the integral parameter, $\lambda$. Dashed horizontal lines show the $\lambda$ levels depicted in Figure 6. Vertical segments have been verified with exact computations as described in section 3.5 .

will be called the isolating block bifurcation diagram for the given masses. The next figure shows the diagram for two choices of the masses. The shaded region shows the parameter values below the critical level $\bar{\lambda}$ for which the inequality is satisfied. The three $\lambda$ values from Figure 6 are indicated by dashed lines.

These plots give some idea of the complexity of the function $W$ and its dependence on the parameters. Nevertheless, with some help from the computer, one can make some rigorous statements about existence of the blocks (see section 3.5). But before taking up these computations, a simpler inequality guaranteeing the existence of infinitesimal isolating blocks will be derived.

As above let $\bar{\lambda}=V(\bar{\theta}, 0)$ be the critical value corresponding to the collinear relative equilibrium with $m_{3}$ between $m_{1}$ and $m_{2}$. Just as in the restricted problem, one can check the inequality (3.24) for $\lambda-\bar{\lambda}<0$ small by means of local calculations at the critical point. The Taylor expansion of $V$ is of the form

$$
V(\bar{\theta}+\delta \theta, \phi)=\bar{\lambda}+\frac{1}{2}\left(\alpha(\delta \theta)^{2}-\beta \phi^{2}\right)+\ldots,
$$


where $\alpha=V_{\theta \theta}(\bar{\theta}, 0)$ and $\beta=V_{\phi \phi}(\bar{\theta}, 0)$. It can be shown that $\alpha$ and $\beta$ are positive for all choices of the masses $m_{i}$.

Setting

$$
\delta \theta=\epsilon \xi, \quad \phi=\epsilon \psi, \quad \lambda=\bar{\lambda}-\epsilon^{2},
$$

inequality (3.24) is approximately

$$
\alpha^{2} \xi^{2}>\bar{\lambda}\left(\alpha \xi^{2}-\beta \psi^{2}\right)+2>\bar{\lambda} \alpha \xi^{2}+2
$$

and the boundary curve of the reduced normalized Hill's region $\mathcal{H}_{1}(h, \omega)=\{(\theta, \phi)$ : $V \geq \lambda\}$ is approximately the hyperbola

$$
\alpha \xi^{2}-\beta \psi^{2}=2
$$

The existence of a constant $\xi$ for which the required inequality holds reduces to $\alpha-\bar{\lambda}>0$ or

$$
V_{\theta \theta}(\bar{\theta}, 0)>V(\bar{\theta}, 0)
$$

It only remains to calculate these two numbers. Of course the values will depend on the masses $m_{i}$ and it turns out that the inequality does not always hold.

Although the use of spherical coordinates leads to conceptually simple formulas, it is not the best choice for calculation. Instead of directly computing $V_{\theta \theta}$ it is better to work with an equivalent quantity in the original Cartesian coordinates of section [3.1.1. Essentially, it is necessary to understand the Hessian of the potential function restricted to the tangent space to the set of normalized collinear configurations. The Hessian at a critical point of a function $U$ on a manifold is a well-defined quadratic form $D^{2} U$ on the tangent space at the critical point. If $c(t)$ is any curve in the manifold passing through the critical point at $t=0$, then

$$
\left.\frac{d}{d t} U(c(t))\right|_{t=0}=0,\left.\quad \frac{d^{2}}{d t^{2}} U(c(t))\right|_{t=0}=D^{2} U(\dot{c}(0), \dot{c}(0)) .
$$

Choose the curve $c(t)=\left(q_{1}(t), q_{2}(t), q_{3}(t)\right)$ in Cartesian coordinates so that for all $t$ the configuration is collinear with center of mass 0 and moment of inertia 1 . Also assume that $c(0)$ is a collinear relative equilibrium. Now if $\bar{c}(t)=(\theta(t), 0)$ is the image curve in $\mathbf{S}^{2}$ under the quotient map of the symmetry group, the equation $U(c(t))=V(\bar{c}(t))$ will hold for all $t$. It follows that the Hessians satisfy

$$
D^{2} U(\dot{c}(0), \dot{c}(0))=D^{2} V(\dot{\bar{c}}(0), \dot{\bar{c}}(0)) \text {. }
$$

If the curve is chosen so that $\dot{\bar{c}}(0)=(\dot{\theta}(0), 0)=(1,0)$, then the right-hand side of this formula will be $V_{\theta \theta}(\bar{\theta}, 0)$.

The construction of the Cartesian curve $c(t)$ requires a small digression. The Hopf map $\bar{h}(x, y)=\left(h_{1}(x, y), h_{2}(x, y),\left|h_{3}(x, y)\right|\right)$ maps the Jacobi configuration variables $(x, y) \in \mathbf{R}^{2 d}$ onto $\mathbf{S}^{2}$. This can be pulled back to the Cartesian variables as follows. Let $q=\left(q_{1}, q_{2}, q_{3}\right) \in \mathbf{R}^{3 d}$. The center of mass equation $m_{1} q_{1}+m_{2} q_{2}+$ $m_{3} q_{3}=0$ defines a $2 d$-dimensional plane, $P$. The linear map (3.3) is an isomorphism $L: P \rightarrow \mathbf{R}^{2 d}$. The composition $f=\bar{h} \circ L: P \rightarrow \mathbf{S}^{2}$ maps the Cartesian variables to the sphere. Once the curve $c(t)=\left(q_{1}(t), q_{2}(t), q_{3}(t)\right)$ has been chosen, then $\bar{c}(t)=f(c(t))$.

Now the important point is that $L$ is an isometry and $\bar{h}$ a similarity with respect to a certain inner product called the "mass metric". The formulas for the norm of 
this inner product in Cartesian and Jacobi coordinates are

$$
\begin{aligned}
\|q\|^{2} & =m_{1}\left|q_{1}\right|^{2}+m_{2}\left|q_{2}\right|^{2}+m_{3}\left|q_{3}\right|^{2}, \\
\|(x, y)\|^{2} & =\mu_{1}|x|^{2}+\mu_{2}|y|^{2},
\end{aligned}
$$

where $|\cdot|$ denotes the ordinary Euclidean norm. It is not difficult to check that these two formulas are related by the map $L$ which is therefore an isometry. Since $\bar{h}$ collapses from dimension $2 d$ to dimension 2 , it cannot really be an isometry or a similarity. However, it does act as a similarity on tangent vectors to $\Sigma$ which are orthogonal to the symmetry group orbits. More precisely, let $(\xi, \eta) \in \Sigma$ and consider a vector $(\hat{u}, \hat{w}) \in T_{(\xi, \eta)} \Sigma$. For simplicity, only the relevant case, where $(\xi, \eta)$ is a collinear configuration and $(\hat{u}, \hat{w})$ is tangent to the submanifold of collinear configurations through $(\xi, \eta)$, will be considered. Note that the derivatives of the functions $h_{i}(x, y)$ are given by

$$
D h_{i}(x, y)(u, w)=2 b_{i}(x, y ; u, w),
$$

where $b_{i}$ are the bilinear functions (3.15). The assumptions on $(\hat{u}, \hat{w})$ imply that $b_{0}=b_{3}=\Omega=0$. It follows that $D \bar{h}(\xi, \eta)(\hat{u}, \hat{w})=2\left(b_{1}, b_{2}, 0\right)$. Then the identity (3.16) gives

$$
|D \bar{h}(\xi, \eta)(\hat{u}, \hat{w})|^{2}=4\left(b_{1}^{2}+b_{2}^{2}\right)=4\|(\hat{u}, \hat{w})\|^{2} .
$$

This proves that $D \bar{h}$ acts as a similarity with scale factor 2 on $(\hat{u}, \hat{w})$.

All this geometry can be applied to give a test for the existence of infinitesimal isolating blocks.

Theorem 3.6. Let $(x, y)$ be a collinear relative equilibrium with $\theta(x, y)=\bar{\theta}$ and $V(\bar{\theta}, 0)=\bar{\lambda}$ and consider subsets $B_{\epsilon}(\xi) \subset \mathcal{M}(h, \omega)$ defined by $\bar{\theta}-\epsilon \xi \leq \bar{\theta} \leq \bar{\theta}+\epsilon \xi$, where $\lambda=\sqrt{2|h| \omega^{2}}=\bar{\lambda}-\epsilon^{2}$. Then there exist $\xi>0, \epsilon_{0}>0$ such that $B_{\epsilon}(\xi)$ is an isolating block for $0<\epsilon<\epsilon_{0}$ provided the following condition holds at the relative equilibrium:

$$
r^{2} \tau>\frac{5}{2} \bar{\lambda}
$$

where $\tau=\frac{m_{1}+m_{2}}{r_{12}^{3}}+\frac{m_{1}+m_{3}}{r_{13}^{3}}+\frac{m_{2}+m_{3}}{r_{23}^{3}}$ and $\bar{\lambda}=\frac{m_{1} m_{2}}{r_{12}}+\frac{m_{1} m_{3}}{r_{13}}+\frac{m_{2} m_{3}}{r_{23}}$.

Proof. It will be shown that (3.25) reduces to the inequality in the theorem. Without loss of generality, one may assume that $(x, y)$ represents a configuration with all three masses on the $x$-axis in $\mathbf{R}^{d}$. Then $q_{i}=\left(x_{i}, 0\right), 0 \in \mathbf{R}^{d-1}$. Choose a curve of collinear configurations $c(t)=\left(q_{1}(t), q_{2}(t), q_{3}(t)\right)$ with $q_{i}(t)=\left(x_{i}(t), 0\right)$ and $x_{i}(0)=x_{i}$. The constraints

$$
\begin{aligned}
& m_{1} x_{1}(t)+m_{2} x_{2}(t)+m_{3} x_{3}(t)=0, \\
& m_{1} x_{1}^{2}(t)+m_{2} x_{2}^{2}(t)+m_{3} x_{3}^{2}(t)=1
\end{aligned}
$$

restrict the curve to an ellipse in $\mathbf{R}^{3 d}$. Finally, if one also requires that

$$
\|\dot{c}(0)\|=\frac{1}{2} \text {, }
$$

then the tangent vector $\dot{c}(0)$ is uniquely determined up to a sign. These conditions on $c(t)$ imply that the image curve $\bar{c}=f(c(t)) \in \mathbf{S}^{2}$ is of the form $(\theta(t), 0)$ with $\theta(0)=\bar{\theta}$ and $\dot{\theta}(0)= \pm 1$. Hence

$$
V_{\theta \theta}(\bar{\theta}, 0)=D^{2} U((\hat{u}, \hat{w}),(\hat{u}, \hat{w})) .
$$

The Hessian of the Newtonian potential on $\Sigma$ in Cartesian coordinates is wellunderstood. Here only the Hessian of the restriction to the collinear configurations 
with $q_{i}=\left(x_{i}, 0\right)$ is needed and it is given by the $3 \times 3$ matrix $H=2 K+U M$, where $U$ is the potential, $I$ is the identity matrix, $M=\operatorname{diag}\left[m_{1}, m_{2}, m_{3}\right]$ and

$$
K=\left[\begin{array}{ccc}
\frac{m_{1} m_{2}}{r_{12}^{3}}+\frac{m_{1} m_{3}}{r_{13}^{3}} & -\frac{m_{1} m_{2}}{r_{12}^{3}} & -\frac{m_{1} m_{3}}{r_{1}^{3}} \\
-\frac{m_{1} m_{2}}{r^{3}} & \frac{m_{1} m_{2}}{r_{12}^{3}}+\frac{m_{2} m_{3}}{r_{23}^{3}} & -\frac{m_{2}^{3} r_{3}}{r_{23}^{3}} \\
-\frac{m_{1}^{3} m_{3}}{r_{13}^{3}} & -\frac{m_{2} m_{3}^{3}}{r_{23}^{3}} & \frac{m_{1} m_{3}}{r_{13}^{3}}+\frac{m_{2} m_{3}}{r_{23}^{3}}
\end{array}\right] .
$$

The mass metric in these coordinates is represented by $M$. The quadratic form $K$ can be put into diagonal form with respect to the inner product represented by $M$. If $d_{1}, d_{2}, d_{3}$ and $v_{1}, v_{2}, v_{3}$ are the eigenvalues and eigenvectors of $M^{-1} K$, then the $v_{i}$ are orthogonal with respect to $M$. If the $v_{i}$ are normalized to be unit vectors, then the matrix of the quadratic form $C$ in this basis is $\operatorname{diag}\left[d_{1}, d_{2}, d_{3}\right]$. Now two of the eigenvectors of $M^{-1} K$ are easily guessed, namely $v_{1}=(1,1,1)$ and $v_{2}=\left(x_{1}, x_{2}, x_{3}\right)$. The first has eigenvalue $d_{1}=0$. For the second one has $K v_{2}=-\left(U_{x_{1}}, U_{x_{2}}, U_{x_{3}}\right)$. Since $q$ is a relative equilbrium, the Cartesian analogue of (3.10) and (3.11) gives $M^{-1} K v_{2}=U v_{2}$. Thus the second eigenvalue of $M^{-1} K$ is $d_{2}=U(q)=V(\bar{\theta}, 0)=\bar{\lambda}$. To find the third note that

$$
\operatorname{tr} M^{-1} K=\tau=\frac{m_{1}+m_{2}}{r_{12}^{3}}+\frac{m_{1}+m_{3}}{r_{13}^{3}}+\frac{m_{2}+m_{3}}{r_{23}^{3}}
$$

and $d_{3}=\tau-\bar{\lambda}$. Since the Hessian was represented by $2 K+U M$, it follows that

$$
D^{2} U\left(v_{3}, v_{3}\right)=2 d_{3}+\bar{\lambda}=2 \tau-\bar{\lambda}
$$

Now the uniquely determined tangent direction to $c(t)$ is orthogonal to both $v_{1}$ and $v_{2}$ with respect to $M$ and so must lie along $v_{3}$. Taking into account the requirement that $\|\dot{c}(0)\|=\frac{1}{2}$ shows that

$$
V_{\theta \theta}(\bar{\theta}, 0)=D^{2} U((\hat{u}, \hat{w}),(\hat{u}, \hat{w}))=\frac{1}{4}(2 \tau-\bar{\lambda})=\frac{1}{2} \tau-\frac{1}{4} \bar{\lambda} .
$$

Substituting this into the inequality (3.25) gives

$$
\tau>\frac{5}{2} \bar{\lambda}
$$

Finally, dropping the normalization of $(x, y)$ gives (3.26).

It will be shown in the next section that inequality (3.26) holds for the majority of mass triples $\left(m_{1}, m_{2}, m_{3}\right)$ but not for all (see Figure 10). Roughly speaking, the infinitesimal blocks exist provided the middle mass, $m_{3}$, is not too large.

The main results of this paper, Theorems 3.5 and 3.6 provide simple tests for existence of isolating blocks near the collinear relative equilibria of the unrestricted three-body problem. If the masses and integrals and the positions of the walls of the set $B$ are chosen so that these inequalities are satisfied, then $B$ will be an isolating block and conclusions about the flow similar to those described for the restricted problem at the end of section 2.4 will follow. First, if $S$ is the maximal invariant set in $B$, then $S$ is a nonempty, compact invariant set in the interior of $B$. Figure 9 shows an isolating block for the equal mass three-body problem on the integral manifold with $\lambda=3.5$. The figure shows an orbit from the invariant set $S$ together with some orbits meeting the walls tangentially, illustrating the convexity condition. In addition there is a transit orbit crossing the block from one wall to the other.

The most important conclusion following from the existence of the isolating blocks concerns the stable and unstable sets $S^{ \pm}$of $S$, characterized as the set of orbits which remain in $B$ for all forward or for all backward times. The fact that 


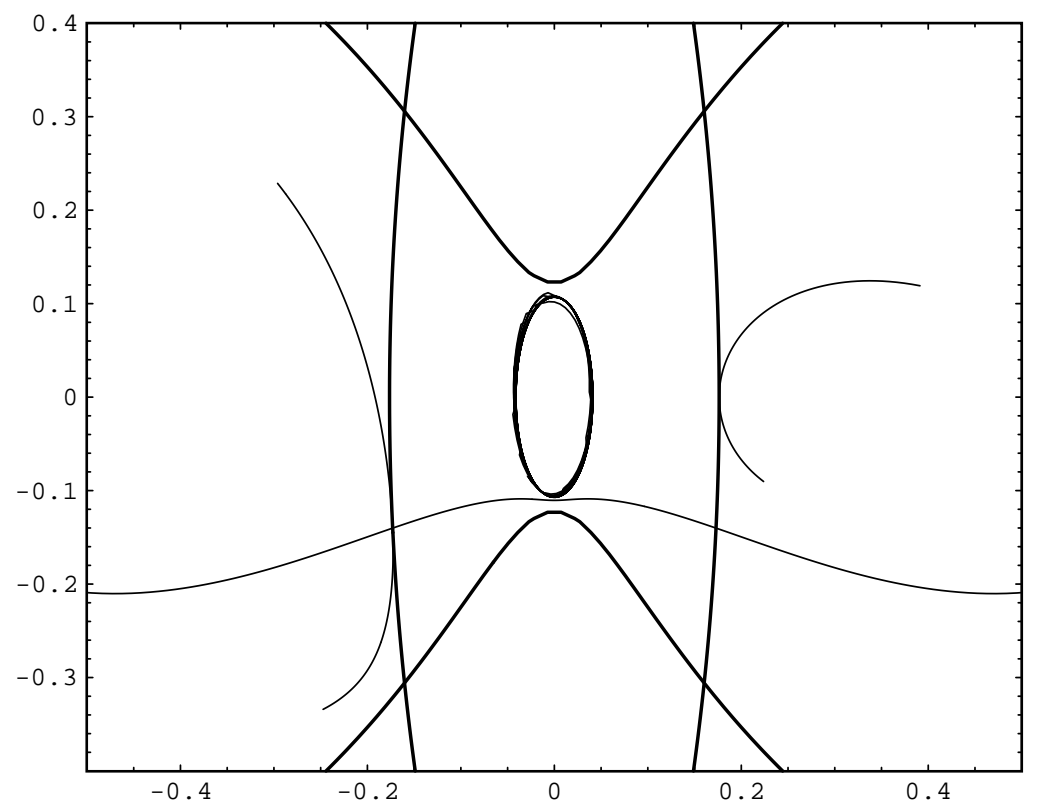

FIGURE 9. An isolating block for the equal mass three-body problem at integral parameter $\lambda=3.5$. The walls of the block are chosen to be $\theta= \pm 20^{\circ}$ which is well within the shaded region of Figure 8 .

the exit set of $B$ is disconnected implies that $S^{ \pm}$are separatrices in the integral manifolds, by the same argument used for the restricted problem. The dimensions of the integral manifolds of the planar and spatial unrestricted problems are 5 and 7, respectively. Thus the separatrices must have dimensions 4 and 6 (at least).

The conclusion about $S$ itself, that it is nonempty and compact, is rather weak, particularly since it was already known that the elliptical Lagrange periodic orbits are contained in it. As in the restricted case, the existence of transit orbits like the one in Figure 9 can be used to show that $S$ carries Cech cohomology in codimension two. This would imply a topological dimension for $S$ of at least 3 in the planar case and 5 in the spatial case, just like the invariant spheres which are known to exist for small perturbations of the relative equilibrium. But rigorously proving the existence of such transit orbits remains an interesting open problem.

3.5. Analysis of the isolating block inequalities. Theorems 3.5 and 3.6 show that isolating blocks for the unrestricted planar and spatial three-body problems exist whenever certain inequalities are satisfied. In this section a method for analyzing these inequalities with the help of rigorous computer algebra computations will be described. Details of these computation will be given in [11].

The infinitesimal isolating block inequality (3.26) can be reduced to an explicit inequality involving the masses as follows. Recall that the collinear relative equilibrium satisfies Euler's fifth degree equation (3.12), where $\rho$ is the distance ratio $r_{13} / r_{12}$. The other distance ratio is $r_{23} / r_{12}=1-\rho$. Then using the normalization 
$r_{12}=1$ one finds

$$
\begin{aligned}
\tau & =\left(m_{1}+m_{2}\right)+\frac{m_{1}+m_{3}}{\rho^{3}}+\frac{m_{2}+m_{3}}{(1-\rho)^{3}}, \\
V(\bar{\theta}, 0) & =\bar{\lambda}=m_{1} m_{2}+\frac{m_{1} m_{3}}{\rho}+\frac{m_{2} m_{3}}{(1-\rho)}, \\
r^{2} & =\frac{1}{m}\left(m_{1} m_{2}+m_{1} m_{3} \rho^{2}+m_{2} m_{3}(1-\rho)^{2}\right) .
\end{aligned}
$$

The boundary of the set of masses where (3.26) holds is given by setting $2 r^{2} \tau-5 \bar{\lambda}=$ 0 . After clearing denominators, this is another polynomial equation for $\rho$. Taking the resultant of this equation and Euler's equation and dropping extraneous positive factors gives the homogeneous polynomial equation $R=0$ for the boundary in the mass space, where $R$ is given by

$$
\begin{aligned}
& 125 m_{1}{ }^{6} m_{2}{ }^{2}-284 m_{1}{ }^{5} m_{2}{ }^{3}+526 m_{1}{ }^{4} m_{2}{ }^{4}-284 m_{1}{ }^{3} m_{2}{ }^{5}+125 m_{1}{ }^{2} m_{2}{ }^{6} \\
& +250 m_{1}{ }^{6} m_{2} m_{3}-978 m_{1}{ }^{5} m_{2}{ }^{2} m_{3}+72 m_{1}{ }^{4} m_{2}{ }^{3} m_{3}+72 m_{1}{ }^{3} m_{2}{ }^{4} m_{3} \\
& -978 m_{1}{ }^{2} m_{2}{ }^{5} m_{3}+250 m_{1} m_{2}{ }^{6} m_{3}+125 m_{1}{ }^{6} m_{3}{ }^{2}-222 m_{1}{ }^{5} m_{2} m_{3}{ }^{2} \\
& -1775 m_{1}{ }^{4} m_{2}{ }^{2} m_{3}{ }^{2}+7560 m_{1}{ }^{3} m_{2}{ }^{3} m_{3}{ }^{2}-1775 m_{1}{ }^{2} m_{2}{ }^{4} m_{3}{ }^{2}-222 m_{1} m_{2}{ }^{5} m_{3}{ }^{2} \\
& +125 m_{2}{ }^{6} m_{3}{ }^{2}-116 m_{1}{ }^{5} m_{3}{ }^{3}+216 m_{1}{ }^{4} m_{2} m_{3}{ }^{3}-2760 m_{1}{ }^{3} m_{2}{ }^{2} m_{3}{ }^{3} \\
& -2760 m_{1}{ }^{2} m_{2}{ }^{3} m_{3}{ }^{3}+216 m_{1} m_{2}{ }^{4} m_{3}{ }^{3}-116 m_{2}{ }^{5} m_{3}{ }^{3}+718 m_{1}{ }^{4} m_{3}{ }^{4} \\
& +1720 m_{1}{ }^{3} m_{2} m_{3}{ }^{4}-1887 m_{1}{ }^{2} m_{2}{ }^{2} m_{3}{ }^{4}+1720 m_{1} m_{2}{ }^{3} m_{3}{ }^{4}+718 m_{2}{ }^{4} m_{3}{ }^{4} \\
& -436 m_{1}{ }^{3} m_{3}{ }^{5}+958 m_{1}{ }^{2} m_{2} m_{3}{ }^{5}+958 m_{1} m_{2}{ }^{2} m_{3}{ }^{5}-436 m_{2}{ }^{3} m_{3}{ }^{5}-83 m_{1}{ }^{2} m_{3}{ }^{6} \\
& -106 m_{1} m_{2} m_{3}{ }^{6}-83 m_{2}{ }^{2} m_{3}{ }^{6}-32 m_{1} m_{3}{ }^{7}-32 m_{2} m_{3}{ }^{7} .
\end{aligned}
$$

This is an eighth-degree algebraic curve in the projective plane determined by the masses. However its intersection with the set of real positive masses turns out to be fairly simple. Moreover, the inequality $R>0$ is equivalent to (3.6) and provides an immediate test for whether a given set of masses admits infinitesimal blocks.

To investigate the curve $R=0$, it is convenient to eliminate one variable by normalizing the masses. Assume that $m_{12}=m_{1}+m_{2}=1$ and introduce two parameters, $u, v \in \mathbf{R}$, such that

$$
m_{1}=\frac{1}{2}-u, \quad m_{2}=\frac{1}{2}+u \quad m_{3}=v, \quad u=\frac{m_{2}-m_{1}}{2 m_{12}}, \quad v=\frac{m_{3}}{m_{12}} .
$$

Then the parameter values corresponding to nonnegative masses are $-\frac{1}{2} \leq u \leq \frac{1}{2}$ and $0 \leq v<\infty$. It is also convenient to replace the distance $\rho$ by a parameter $z=\frac{1}{2}-\rho$ so that the mutual distances become

$$
r_{12}=1, \quad r_{13}=\frac{1}{2}-z, \quad r_{23}=\frac{1}{2}+z .
$$

The distance $\rho$ satisfies $0<\rho<1$ and so $z$ satisfies $-\frac{1}{2}<z<\frac{1}{2}$. In the new variables, the equation $R=0$ becomes

$$
\begin{aligned}
& 13+8 u^{2}+656 u^{4}-8960 u^{6}+21504 u^{8}-164 v \\
& +1056 u^{2} v+15424 u^{4}-68096 u^{6} v+954 v^{2}-17600 u^{2} v^{2} \\
& +128800 u^{4} v^{2}-166656 u^{6} v^{2}-2660 v^{3}+19168 u^{2} v^{3} \\
& -63808 u^{4} v^{3}+2989 v^{4}+49560 u^{2} v^{4}-62256 u^{4} v^{4}+2088 v^{5} \\
& -36256 u^{2} v^{5}-1088 v^{6}-960 u^{2} v^{6}-512 v^{7}=0 .
\end{aligned}
$$




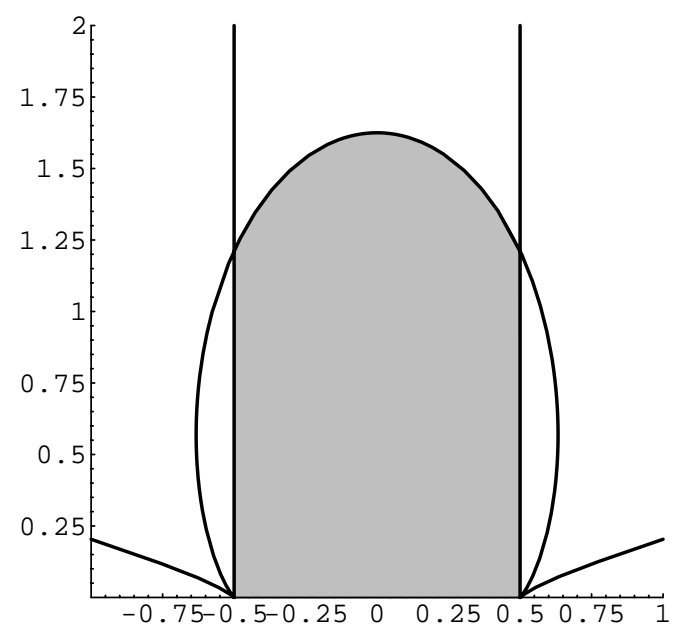

Figure 10. Masses for which infinitesimal isolating blocks $B_{\epsilon}$ exist lie below the $\Omega$ curve in the vertical strip. The variables are $u=\frac{m_{2}-m_{1}}{2\left(m_{1}+m_{2}\right)}$ and $v=\frac{m_{3}}{m_{1}+m_{2}}$.

Figure 10] shows a portion of the curve shaped like the Greek letter $\Omega$. The rounded central part connecting the cusps at $\left( \pm \frac{1}{2}, 0\right)$ corresponds to parameters $-\frac{1}{2}<z<\frac{1}{2}$. The vertical lines at $u= \pm \frac{1}{2}$ mark the boundaries of the strip where the masses are nonnegative. Evidently, the equation $2 r^{2} \tau-5 \bar{\lambda}=0$ determines a convex curve which cuts the strip into two parts. The shaded part of the strip below the curve is where the isolating block inequality $r^{2} \tau>\frac{5}{2} \bar{\lambda}$ holds. In particular, it holds whenever the value of $v=\frac{m_{3}}{m_{1}+m_{2}}$ is less than a certain constant $v_{0} \approx 1.21$. For all masses corresponding to this region, Theorem 3.6 shows that the isolating blocks $B_{\epsilon}$ can be constructed for all sufficiently small $\epsilon$.

Some of the apparent properties of the omega curve can be rigorously verified using algebraic computations. The key to this is the remarkable fact that this degree-eight algebraic curve is rational, i.e, it admits a parametrization $u(z), v(z)$ by rational functions giving the mass variables as functions of the shape variable $z$. Moreover, these functions have integer coefficients. The parametrizing functions $u(z), v(z)$ can be analyzed using Sturm's algorithm. Recall that Sturm's algorithm gives an effective count of the number of real roots of a real polynomial in any interval [13. The algorithm involves computing a sequence of polynomials and checking the difference in the numbers of sign changes of the sequence at the two endpoints of the interval in question. If the polynomials have integer or rational coefficients and if the endpoints of the interval are also rational, these computations can all be carried out exactly by computer.

It is possible to establish bounds for $u(z)$ and $v(z)$ by showing that expressions of the form $u(z)-L$ or $v(z)-L$ have no real roots. Similarly one can determine the signs of the first two derivatives of $u(z), v(z)$. Using this method one can show that the part of the omega curve in the strip $-\frac{1}{2} \leq u \leq \frac{1}{2}$ is the graph of a concave function $v=\psi(u)$. This function has a maximum value of $\frac{13}{8}$ when $u=0$ and reaches a minimum value $v_{0}>1.21$ at the edges of the strip. 
The more complicated inequalities (3.23) and (3.24) can be used to study the existence of larger isolating blocks which extend far from the relative equilibrium in both the configuration space and the parameter space. For fixed masses, the problem is to find the isolating block bifurcation diagram (see Figure 8). This amounts to finding the parameters $(c, \lambda)$ for which inequality (3.24) holds along the $\operatorname{arc} \theta=c$. Note that the inequality can be written in the form

$$
\lambda^{2}>V^{2}(c, \phi)-V_{\theta}^{2}(c, \phi) .
$$

By Theorem 3.5, this is required to hold for all $\phi$ such that $(c, \phi)$ is in the reduced Hill's region $\tilde{\mathcal{H}}_{1}(\lambda)$. However it holds automatically outside the Hill's region. For each fixed $c \in\left[c_{1}, c_{2}\right]$ choose an interval $\phi_{1}(c) \leq \phi \leq \phi_{2}(c)$ large enough to contain the part of the arc $\theta=c$ in all the Hill's regions $\mathcal{H}(\lambda), \lambda_{0} \leq \lambda \leq \bar{\lambda}$. Let $W(c)=$ $\max \left(V^{2}(c, \phi)-V_{\theta}^{2}(c, \phi)\right)$, where the maximum is taken over the chosen interval. Then $W(c)>0$ is a continuous function and the lower boundary curve of the isolating block bifurcation diagram is given by

$$
\lambda=\sqrt{W(c)} .
$$

The shaded region in the diagrams is the set $\sqrt{W(c)} \leq \lambda \leq \bar{\lambda}$, where $\bar{\lambda}$ is the level of the collinear relative equilibrium. For each fixed $c$ it is easy to estimate $W(c)$ numerically, for example by simply evaluating $V^{2}(c, \phi)-V_{\theta}^{2}(c, \phi)$ at many values of $\phi$. This is essentially how the bifurcation diagrams in Figure 8 were produced. In fact, an equivalent computation in mutual distance coordinates was performed. Using (3.23) instead of (3.24), it is required to check the inequality

$$
\lambda^{2}>U^{2}-\frac{1}{4} \tilde{D} U^{2}
$$

along the $\operatorname{arc} \theta=c$ in the sphere of unit moment of inertia. The functions $U$ and $\tilde{D} U$ are rational functions of the mutual distances. Fixing a value $\theta=c$ is equivalent to setting

$$
h_{2}-\tan (c) h_{1}=0
$$

where $h_{i}$ are given by (3.7). This becomes a polynomial in mutual distance coordinates. Finally, the moment of inertia is another polynomial in the $r_{i j}$. Instead of working on the sphere one can multiply $U$ and $\tilde{D} U$ by appropriate powers of the moment of inertia to obtain homogeneous rational functions of degree 0 . Then one can use the simpler normalization $r_{12}=1$ instead.

The computations will be restricted to a certain triangle $T$ in the $\left(r_{13}, r_{23}\right)$ plane. For the masses considered here the triangle $0 \leq r_{i j} \leq 1$ and $1 \leq r_{13}+r_{23}$ can be used, but for other choices of masses, it is necessary to use a slightly smaller one (see 11 for details). This triangle covers most of the configurations relevant to the study of isolating blocks near $L_{2}$. The curves $h_{2}-\tan (c) h_{1}=0$ in $T$ turn out to be branches of hyperbolas which cross the level curves of the potential monotonically (see Figure 11] (left)). Suppose that the part of the hyperbola $h_{2}-\tan (c) h_{1}=0$ which lies in $T$ crosses all of the level curves $U=\lambda$ for $\lambda_{0} \leq \lambda \leq \bar{\lambda}$. Then the maximum of $U^{2}-\frac{1}{4} \tilde{D} U^{2}$ along this curve equals $W(c)$ provided this maximum exceeds $\lambda_{0}$. Thus one can estimate $W(c)$ numerically just by evaluation of rational functions at many points on the part of the hyperbola in $T$.

If the masses $m_{i}$ and the values of $\tan (c)$ and $\lambda$ are rational numbers, or at least algebraic numbers which are exactly representable in the computer, then this approach can be combined with Sturm's algorithm to provide a rigorous test for whether given parameters $(c, \lambda)$ are in the shaded region of the isolating block 

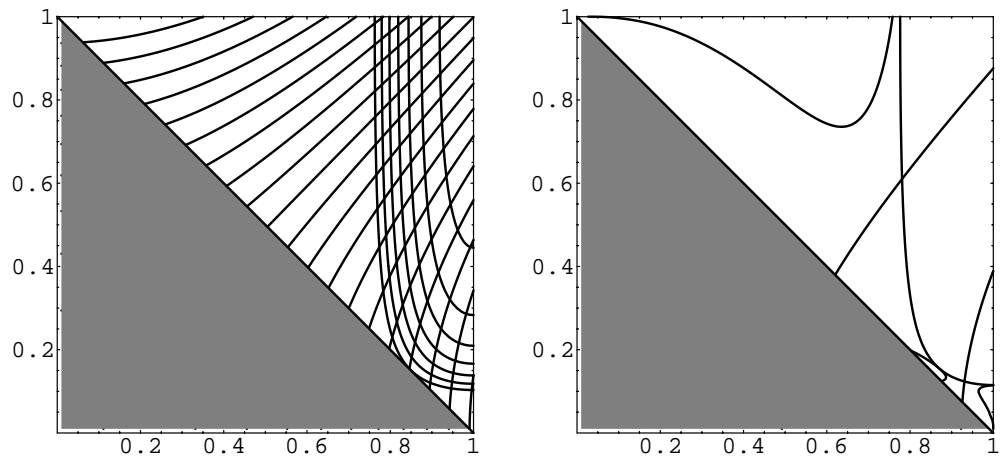

FiguRE 11. Triangle of mutual distances where the isolating block inequality can be analyzed. On the left, level curves of $U$, and $h_{2} / h_{1}$ are shown for $m_{1}=81, m_{2}=1, m_{3}=0.01$. On the right, the curves $U=\lambda, \lambda^{2}=U^{2}-\frac{1}{4} \tilde{D} U^{2}, h_{2} / h_{1}=\tan 7^{\circ}$ and $h_{2} / h_{1}=$ $\tan 10.5^{\circ}$ are shown for $\lambda=81.797$.

bifurcation diagram or not. To reduce the problem to a question about a polynomial in one variable, fix the parameters $\tan (c)$ and $\lambda$ and then eliminate one of the mutual distances $r_{13}$ or $r_{23}$ between the two equations $\lambda^{2}=U^{2}-\frac{1}{4} \tilde{D} U^{2}$ and $h_{2}-\tan (c) h_{1}=0$. The result is a single polynomial equation $R=0$ for the remaining mutual distance with coefficients which are integer polynomials in the masses and the parameters. If this polynomial has no real roots on an appropriate interval and if $\lambda^{2}>U^{2}-\frac{1}{4} \tilde{D} U^{2}$ holds at one endpoint, then it must hold all along the curve as required. This can be rigorously checked with Sturm's algorithm. The geometrical meaning of the test is indicated on the right side of Figure 11 which shows hyperbolic arcs corresponding to two particular values of $c$ as well as the curves where $U=\lambda$ and $\lambda^{2}=U^{2}-\frac{1}{4} \tilde{D} U^{2}$ for a particular choice of $\lambda$. The fact that the arcs do not cross the latter curve is proved by showing that the one-variable polynomial $R$ obtained by elimination has no real roots on the relevant interval.

Unfortunately, the polynomials involved are so complicated that these computations can be carried out only after fixing the masses and parameters. Although this does provide a rigorous method for verifying that the isolating block inequalities hold in particular cases, it does not allow one to understand the whole curve $\lambda=\sqrt{W(c)}$ or to study how the curve changes as the masses change.

\section{ACKNOWLEDGMENTS}

Thanks to Richard Montgomery for some useful information about the Hopf map and to the National Science Foundation for supporting this research under grant DMS 0200992.

\section{REFERENCES}

1. D. Appleyard, thesis, University of Wisconsin, (1968).

2. E. Belbruno and J. Miller, Sun-perturbed earth-to-moon transfers with ballistic capture, Jour. of Guidance, Control and Dynamics, 16 (1993) 770-775.

3. A. Albouy and A. Chenciner, Le probléme des $n$ corps et les distances mutuelles, Inv. Math., 131 (1998) 151-184. MR 98m:70017 
4. C. C. Conley, Low energy transit orbits in the restricted three-body problem, SIAM J. Appl. Math., 16, 4 (1968) 732-746. MR 38:1856

5. C.C. Conley, Isolated Invariant Sets and the Morse Index, CBMS Regional Conference Series, 38, American Mathematical Society (1978). MR 80c:58009

6. C. C. Conley and R. W. Easton, Isolated invariant sets and isolating blocks, Trans. AMS, 158, 1 (1971) 35-60. MR 43:5551

7. R. W. Easton, Existence of invariant sets inside a submanifold convex to the flow, JDE, 7 (1970) 54-68. MR 40:2996

8. R. W. Easton, Some qualitative aspects of three-body flow, in Dynamical Systems, vol. 2, An International Symposium, Academic Press (1976) 1-6. MR 58:32196]

9. W. S. Koon, et al., Constructing a low energy transfer between Jovian moons, in Celestial Mechanics, Contemporary Mathematics, vol. 292, American Mathematical Society (2002) 129-146. MR 2002m:70040]

10. J. L. Lagrange, Ouvres, vol 6, 272.

11. R. Moeckel, Sturm's algorithm and isolating blocks, to appear in JSC.

12. C. Simo, et al., Dynamics and Mission Design Near Libration Points, vols. I, III, World Scientific (2001). MR 2003c:70041a MR 2003c:70041c

13. B. L. van der Waerden, Modern Algebra, Ungar, New York (1949). MR 10:587b

14. A. Wintner, The Analytical Foundations of Celestial Mechanics, Princeton Math. Series 5, Princeton University Press, Princeton, NJ (1941). MR 3:215b

School of Mathematics, University of Minnesota, Minneapolis, Minnesota 55455

E-mail address: rick@math.umn.edu 